\title{
Assigning Product Ions from Complex MS/MS Spectra: The Importance of Mass Uncertainty and Resolving Power
}

\author{
Lekha Sleno and Dietrich A. Volmer \\ Institute for Marine Biosciences, National Research Council, Halifax, Nova Scotia, Canada
}

\author{
Alan G. Marshall \\ National High Magnetic Field Laboratory, Florida State University, Tallahassee, Florida, USA
}

This study offers a unique insight into the mass accuracy and resolving power requirements in MS/MS analyses of complex product ion spectra. In the examples presented here, accurate mass assignments were often difficult because of multiple isobaric interferences and centroid mass shifts. The question then arose whether the resolving power of a medium-resolution quadrupole time-of flight (QqTOF) is sufficient or high-resolution Fourier-transform ion cyclotron resonance (FT-ICR) is required for unambiguous assignments of elemental compositions. For the comparison, two paralytic shellfish poisons (PSP), saxitoxin (STX) and neosaxitoxin (NEO), with molecular weights of 299 and $315 \mathrm{~g} \cdot \mathrm{mol}^{-1}$, respectively, were chosen because of the high peak density in their MS/MS spectra. The assessment of QqTOF collision-induced dissociation spectra and FT-ICR infrared multiphoton dissociation spectra revealed that several intrinsic dissociation pathways leading to isobaric fragment ions could not be resolved with the QqTOF instrument and required FT-ICR to distinguish very close mass differences. The second major source of interferences was $M+1$ species originating from coactivated ${ }^{13} \mathrm{C}^{12} \mathrm{C}_{\mathrm{c}-1}$ ion contributions of the protonated molecules of the PSPs. The problem in QqTOF MS results from internal mass calibration when the $\mathrm{MH}^{+}$ions of analyte and mass calibrant are activated at the same time in the collision or trapping cell. Although FT-ICR MS readily resolved these interfering species, the QqTOF did not provide resolving power $>20,000$ (full width at half maximum) required to separate most isobaric species. We were able to develop a semi-internal QqTOF calibration technique that activated only the isolated ${ }^{12} \mathrm{C}$ isotope species of the protonated molecules, thus reducing the $M+1$ interferences significantly. In terms of overall automated elemental formulas assignment, FT-ICR MS achieved the first formula hit for $100 \%$ of the product ions, whereas the QqTOF MS hit rate was only 56 and $65 \%$ for STX and NEO product ions, respectively. External mass calibration from commercial FT-ICR and QqTOF instruments gave similar results. (J Am Soc Mass Spectrom 2005, 16, 183-198) @ 2004 American Society for Mass Spectrometry

$\mathrm{M}$ ass-based structural identification of drugs and their metabolites frequently employs combinations of collision-induced dissociation (CID) analyses on triple quadrupole and ion trap mass analyzers. These methods often provide adequate information for determining the sites of modification for structural analogs [1-5]. In those cases, however, in which the variety of fragment ions is limited and the spectra do not allow unambiguous structure assignments, tentatively identified structures are often further examined by high-resolution mass spectrometry, from which elemental compositions can be obtained. In these experiments, it is required to measure the mass-tocharge ratios with the smallest mass uncertainties possi-

Published online December 13, 2004

Address reprint requests to Dr. D. A. Volmer, Institute for Marine Biosciences, National Research Council, 1411 Oxford Street, Halifax, Nova Scotia B3H 3Z1, Canada. E-mail: Dietrich.Volmer@nrc-cnrc.gc.ca ble, in particular if the methodologies are part of routine structure identification protocols. Ideally, unattended and automated analytical routines with sufficient resolution and mass accuracy would yield a single empirical formula for every compound analyzed. In most structural elucidation studies, quadrupole time-of-flight (QqTOF) and, increasingly, Fourier-transform ion cyclotron resonance (FT-ICR) mass spectrometers enable exact mass measurements of protonated molecules as well as of their MS/MS product ions [6-11]. Sometimes lowresolution mass spectrometers can be used, when additional information about the atomic composition is available. For example, Fiehn and coworkers [12] recently used gas chromatography/low-resolution quadrupole mass spectrometry to determine elemental formulas of unknown plant metabolites. The authors limited the range of possible formulas by combining the measured masses with isotope ratios.

The number of possible empirical formulas calcu- 


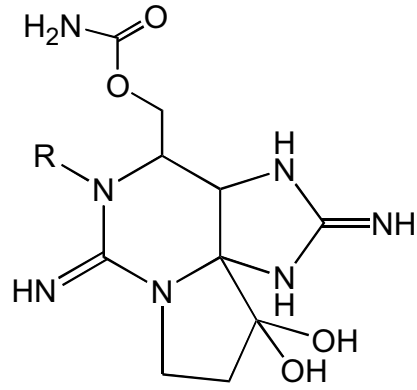

$$
\begin{array}{ll}
\text { Saxitoxin } & \mathrm{R}=\mathrm{H} \\
\text { Neosaxitoxin } & \mathrm{R}=\mathrm{OH}
\end{array}
$$

Scheme 1. Chemical structures of saxitoxin (STX) and 1-Nhydroxysaxitoxin (neosaxitoxin, NEO).

lated from the data decreases rapidly with increasing mass accuracy. In the absence of a unique empirical formula, analysis can be highly ambiguous. The certainty of mass measurements is the topic of the present article.

TOF instruments are often described as having the capability of allowing "exact" mass measurements of small molecules with uncertainties of less than $10 \mathrm{ppm}$ in regular MS mode. Several published reports on mass uncertainties for TOF and QqTOF instruments claim mass accuracies very close to those seen with FT-ICR systems [7, 13-15]. In fact, in the present study, we shall see that many of our measured $\mathrm{m} / \mathrm{z}$ values on the QqTOF instrument can be exceptionally accurate. It is hard to argue with these numbers, but then, such accuracy requires mass precision and accuracy to within as little as $1 \%$ of the TOF mass spectral peak full width at half-maximum peak height. Thus, accurate $\mathrm{m} / \mathrm{z}$ measurement capability is possible only if the mass analyzer can resolve adjacent peaks in very complex samples; e.g., those encountered by researchers in the petroleum industry [16] and by environmental scientists dealing with humic and fulvic acids [17], as well as any sample for which large background signals obscure the measured ions of interest.

Other examples of such "cluttered" mass spectra are collision-induced dissociation (CID) spectra of molecules having a large number of functional groups and the possibility for extensive resonance stabilization of a wide variety of ions formed in competing low-energy fragmentation pathways. We have recently described such an example, observed in the triple-quadrupole CID analysis of protonated molecules of paralytic shellfish poisoning (PSP) toxins (Scheme 1) [18]. Not only did the spectra exhibit an unusually "rich" variety of product ions but they also showed numerous isobaric species as well as interfering $M+1$ contributions from ${ }^{13} \mathrm{C}^{12} \mathrm{C}_{\mathrm{c}-1}$ species. The FT-ICR instrument employed for exact mass measurements in that study was able to resolve all of those species and no false $\mathrm{m} / \mathrm{z}$ assignments from overlapping peaks with resulting mass centroid shifts were observed. The unique complexity of those spectra introduced an interesting example to prompt an instrumental comparison between high-resolution FTICR and medium-resolution QqTOF mass spectrometry. Several such comparisons have recently been published for MS/MS spectra [19-21]. For example, Thompson et al. compared [19] mass accuracies for fluconazole fragment ions based on external calibration sustained off-resonance irradiation (SORI) [23] CID with a 4.7 tesla FT-ICR and a one-point lock-mass corrected (based on the $\mathrm{MH}^{+}$ion) CID external calibration curve for a QqTOF instrument. The mass uncertainties were on average $\pm 0.7 \mathrm{ppm}$ for FT-ICR and \pm 2.9 $\mathrm{ppm}$ for QqTOF experiments. Except for one peak in the QqTOF MS/MS spectrum, the peaks for the 13 fragment ions in the MS/MS spectra were not distorted and the experimentally observed QqTOF resolution of 3200 FWHM (full-width at half maximum; $m / \Delta m$, in which $\Delta m=$ FWHM) was sufficient to resolve all peaks. Also, Hau and coworkers demonstrated [20] an excellent comparison of QqTOF and FT-ICR MS/MS spectra for a group of eight low-molecular weight aza-heterocycles. The authors recalibrated the externally calibrated MS/MS spectra by merging the individual analyte spectra with the MS/MS spectrum of a reference compound acquired under the same conditions. As a result, they obtained an average mass uncertainty of approximately $30 \mathrm{ppm}$ with the QqTOF for 51 fragment ions, whereas the FT-ICR error was always less than $1.5 \mathrm{ppm}$. More important, true elemental composition as the "first hit" was established for only about $70 \%$ of all fragment ions with the QqTOF, whereas FT-ICR revealed the correct formula with a 100\% hit rate.

Finally, the precision of any mass measurement (i.e., the rms deviation from a large number of repeated measurements) is related to the peak shape, the signal-to-noise ratio, and the square root of the number of data points per peak width for a single measurement [22]. Thus, high mass measurement accuracy with a TOF mass analyzer typically requires very high signal-to-noise ratio.

The aim of the present work, and the novel aspect to justify yet another instrumental comparison, was to investigate particularly complex CID spectra for which accurate mass assignments are much more difficult because of isobaric interferences and thus, potentially, overlapping peaks and shifts in the centroid masses. Except for isomeric ions, isobaric signals will always lead to inhomogeneous peak broadening in the spectra due to the different mass defects of the elements, if the mass spectrometer has insufficiently high resolving power. The question then arises whether or not the resolving power of QqTOF is sufficient or FT-ICR is required for unambiguous identifications. Specifically, for the exact measurement of PSP product ions in MS/MS spectra, we consider: (1) comparison of different calibration techniques for MS/MS on QqTOF and FT-ICR; (2) detailed study of regions of spectral interferences in the MS/MS spectra and the influence of 

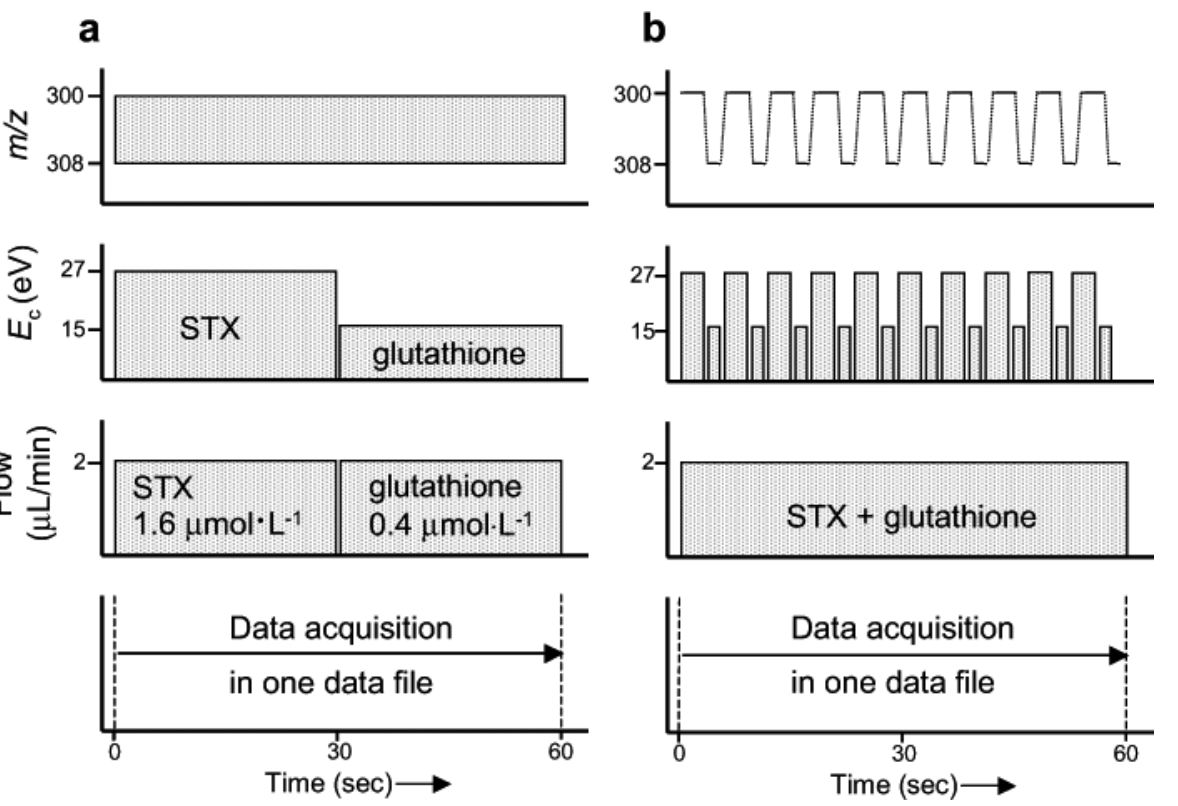

Scheme 2. Two different mass calibration protocols were used for QqTOF experiments (shown for STX). (a) Simultaneous activation of the $\mathrm{m} / \mathrm{z}$ range 300-308 with sequential infusion of STX and glutathione; (b) alternating monoisotopic activation of $\mathrm{m} / \mathrm{z} 300$ (STX) and $\mathrm{m} / \mathrm{z} 308$ (glutathione) while continuously infusing PSP + glutathione.

resolving power; and (3) the assessment of the ability to fully automate assignment of elemental compositions to MS/MS fragment ions. Although we perform CID for QqTOF and infrared multiphoton dissociation (IRMPD) for FT-ICR MS/MS experiments, both low-energy fragmentation methods typically act by slow heating of an ion so as to cleave the weakest bond, so that both methods tend to generate similar products $[23,24]$.

\section{Experimental}

\section{Chemicals and Standard Solutions}

Reference standard solutions of saxitoxin $\left(65 \mu \mathrm{mol} \cdot \mathrm{L}^{-1}\right.$ in $\left.3 \cdot 10^{-3} \mathrm{M} \mathrm{HCl}\right)$ and neosaxitoxin $\left(65 \mu \mathrm{mol} \cdot \mathrm{L}^{-1}\right.$ in $3 \cdot 10^{-3} \mathrm{M}$ $\mathrm{HCl})$ were obtained through IMB's Certified Reference Materials Program (Halifax, NS, Canada) and were diluted 40-fold in 50:50 ( $\mathrm{vol} / \mathrm{vol})$ methanol/water $(+0.1 \%$ formic acid) prior to infusion into the mass spectrometers. Formic acid, $\mathrm{N}_{\alpha}$-acetyl-Arg, Lys-Leu, Phe-Gly-Gly-Phe, glutathione ( $\gamma$-Glu-Cys-Gly) and $\left[\mathrm{Glu}^{1}\right]$-fibrinopeptide were purchased from Sigma-Aldrich (Mississauga, ON, Canada). Methanol (Caledon, Georgetown, ON, Canada) and Milli-Q organic free water (Millipore, Bedford, MA) were used as solvents.

\section{Quadrupole Time-of-Flight MS/MS}

$Q_{1} q_{2}$ TOF experiments were performed with an MDS Sciex (Concord, ON, Canada) QSTAR Pulsar $i$ mass spectrometer. The instrument was operated under the following conditions: spray voltage, $5 \mathrm{kV}$; declustering potentials (DP1, DP2), 30 and $40 \mathrm{~V}$, respectively; collision gas, Ar (collision gas pressure, 6 [arbitrary units]).
Mass calibration for MS/MS was performed with glutathione as internal mass calibrant. Two different procedures were compared. Initially, the $\mathrm{Q}_{1}$ resolution was modified to allow transfer of $\mathrm{MH}^{+}$ions of both PSP and glutathione into $\mathrm{q}_{2}(\mathrm{~m} / \mathrm{z}$ 300-308 or 308-316), for concurrent activation of all ions in that mass range. Because of the different activation energies required for equivalent dissociation yields for $m / z$ 300/316 (STX/NEO) and 308 (glutathione), simultaneous activation was not possible with the chosen mass calibrant. Instead, the $\mathrm{MH}^{+}$ions were introduced sequentially (30 s PSP at $27 \mathrm{eV}$ and then $30 \mathrm{~s}$ glutathione at $15 \mathrm{eV}$; with all other experimental parameters identical), but the data acquisition was never stopped during the process. The acquisition sequence is illustrated in Scheme $\mathbf{2 a}$. This procedure closely resembled true internal mass calibration and, importantly, exhibited all of the characteristic features essential to the discussion in this paper (vide infra). The second method involved monoisotopic selection and rapid "back-and-forth" switching between $\mathrm{m} / \mathrm{z}$ 300/316 and 308 in $\mathrm{Q}_{1}$, followed by CID in $\mathrm{q}_{2}$. During these scans, the PSPs $\mathrm{MH}^{+}$ions were activated at $27 \mathrm{eV}$ activation energy for $4 \mathrm{~s}$, followed by activation of gluthathione's $\mathrm{MH}^{+}$ion for $2 \mathrm{~s}$ at $15 \mathrm{eV}$, then PSP again and so forth (Scheme $\mathbf{2 b}$ ). In other words, the MS/MS scans were acquired in repeating cycles, for the analyte first and then the calibrant. The scans were continuously acquired into one data file, allowing internal mass recalibration of the data. For both calibration routines, a simple three-point internal calibration curve based on three glutathione peaks $(m / z$ 76.02209, 162.02194, and 308.09108) was used. All QqTOF spectra were generated by averaging the entire data set over the 
$60 \mathrm{~s}$ acquisition period. From the same data without the 3-point calibration correction, external calibration data was used for a separate comparison. Use of closelyspaced individual two-mass calibration points of slightly higher and lower masses for every PSP product ion did not improve the overall mass accuracy.

\section{IRMPD FT-ICR MS/MS}

FT-ICR measurements for the internal calibration experiments were performed with a home-built 9.4 tesla FT-ICR instrument [25] equipped for external octopole ion accumulation [26]. Sample and calibration solutions were introduced at $400 \mathrm{~nL} \cdot \mathrm{min}^{-1}$ from separate sprayers of a dual electrospray ionization (ESI) source [27]. Ions were transferred through a Chait-style atmosphere-to-vacuum interface [28] and accumulated in a linear octopole, modified for improved ion ejection along the z-axis [29]. Mass-selected calibrant and sample ions were individually accumulated for 5 and $1 \mathrm{~s}$, respectively. All ions were then transferred through an octopole ion guide to the ICR cell. Hanning apodization and one zero-fill were applied to all data prior to the fast Fourier-transform and magnitude calculation [30] and frequency-to- $m / z$ conversion [31, 32]. For infrared multiphoton dissociation (IRMPD), product ion data were internally calibrated by tailoring the stored-waveform inverse Fourier transform (SWIFT) [33, 34] waveform to isolate precursor (STX, $\mathrm{m} / \mathrm{z} 300 ; \mathrm{NEO}, \mathrm{m} / \mathrm{z}$ 316) and Glu-Fib $[\mathrm{M}+2 \mathrm{H}]^{2+}(\mathrm{m} / \mathrm{z} 785.8)$ ions, followed by irradiation with an off-axis $40 \mathrm{~W} \mathrm{CO} 2$ laser (Synrad E48-2-115, Bothell, WA): $10.6 \mu \mathrm{m}$ wavelength, $150 \mathrm{~ms} ; 90 \%$ laser power, fitted with a beam expander. Glu-Fib product ions of $\mathrm{m} / \mathrm{z} 175.11893\left(\mathrm{y}_{1}\right), 246.15607\left(\mathrm{y}_{2}\right)$, and 333.18807 $\left(y_{3}\right)$ were used for calibration. The generated product ions were then subjected to chirp excitation and direct-mode broadband detection. Spectra represent the sum of 50 scans of $150 \mathrm{~ms}$ each (2 M data). For elemental formula assignments of product ions in IRMPD, the atom constraints for $\mathrm{C}, \mathrm{H}, \mathrm{N}$, and $\mathrm{O}$ were set to the respective number of atoms in the precursor ion molecules $\left(\mathrm{C}_{10} \mathrm{H}_{18} \mathrm{~N}_{7} \mathrm{O}_{4}\right.$ for STX and $\mathrm{C}_{10} \mathrm{H}_{18} \mathrm{~N}_{7} \mathrm{O}_{5}$ for NEO, respectively). All calculations were performed with the MIDAS Molecular Formula Calculator (NHMFL, Tallahassee, FL).

FT-ICR experiments for the external calibration experiments were conducted with a 7 tesla FT-ICR mass spectrometer (Ionspec, Lake Forest, CA). The sample solutions $\left(1.6 \mu \mathrm{mol} \cdot \mathrm{L}^{-1}\right)$ were introduced at $2 \mu \mathrm{L} \cdot \mathrm{min}^{-1}$ into the electrospray source (needle potential, $3.8 \mathrm{kV}$ ). The cone voltage was set to $30 \mathrm{~V}$ and the extractor to $5 \mathrm{~V}$. A hexapole collection period of $1000 \mathrm{~ms}$ was used with an offset voltage of $1 \mathrm{~V}$. Chirp excitation was performed at $140 \mathrm{~V}$ for $4 \mathrm{~ms}$ (range $\mathrm{m} / \mathrm{z}$ 100-2500). IRMPD was conducted with a $25 \mathrm{~W} \mathrm{CO}_{2}$ laser. For STX, either a $50 \%$ laser power setting with a $1400 \mathrm{~ms}$ pulse or $100 \%$ for $500 \mathrm{~ms}$ pulse duration was used (see Table 2a and b for details). The precursor selection was achieved with an arbitrary waveform generator $( \pm 1.5 \mathrm{Da}$ window). For NEO, the laser power was set to 80 or $100 \%$ for $500 \mathrm{~ms}$ pulse duration. The precursor at $\mathrm{m} / \mathrm{z} 316$ was initially isolated with an arbitrary waveform generator at $\pm 4 \mathrm{Da}$, but because of isobars at $\mathrm{m} / \mathrm{z} 316$, an ion isolation pulse was applied to narrow the window to $\pm 0.184 \mathrm{Da}$. The experiment-to-experiment precision of mass measurement was equal or less than $\pm 0.1 \mathrm{mDa}$ in all internal (vide supra) and external calibration experiments.

\section{Results and Discussion}

This study illustrates the importance of concurrent high mass spectral resolution and accurate mass assignment capability for determining elemental compositions from complex product ion spectra from MS/MS of electrosprayed PSP toxins. While medium resolution tandem mass spectrometers such as QqTOF instruments can generate excellent mass accuracy (with less than $10 \mathrm{ppm}$ uncertainty in MS/MS experiments) with resolving power up to 10,000 (FWHM), the situation becomes more difficult when these instruments are applied to dense MS/MS spectra, in which interferences lead to overlapping signals that require higher resolving power. Such interferences may originate from: (1) multiple isobaric species formed through competitive fragmentation reactions after ion activation of $\mathrm{MH}^{+}$ions; (2) overlap of analyte and internal calibrant product ion signals; (3) or $\mathrm{M}+1$ contributions, when both an all- ${ }^{12} \mathrm{C}$ precursor as well a ${ }^{13} \mathrm{C}^{12} \mathrm{C}_{\mathrm{c}-1}$ isotopomer are simultaneously activated in MS/MS experiments. The latter situation can happen when an analyte and a mass calibrant substance of similar molecular weight are activated in the collision or trapping cell at the same time to obtain a composite product ion spectrum for internal calibration purposes. To a lesser extent, $\mathrm{M}+1$ interferences can be expected when the precursor ion selection in $\mathrm{Q}_{1}$ of a QqTOF instrument is not narrow enough to completely isolate the ${ }^{12} \mathrm{C}_{\mathrm{c}}$ species of the analyte from its ${ }^{13} \mathrm{C}^{12} \mathrm{C}_{\mathrm{c}-1}$ isotopomer.

The present article illustrates an instrumental comparison between high-resolution FT-ICR and medium-resolution QqTOF mass spectrometry. Two PSP toxins, saxitoxin (STX) and 1-N-hydroxysaxitoxin (Neosaxitoxin, NEO) (Scheme 1), were chosen as test molecules. The large number of functional groups within a rather small skeletal structure of the two compounds and the possibility for resonance stabilization of fragment ions after dissociation lead to unusually rich product ion mass spectra [18]. This complexity is illustrated in Figure 1, showing QqTOF CID spectra of the $\mathrm{MH}^{+}$ions of STX and NEO. The two compounds exhibited a large number of different product ion species of significant abundance throughout the entire mass range. Not surprisingly, the spectra appear quite similar to the triplequadrupole MS/MS spectra reported before, as the collision conditions in $\mathrm{q}_{2}$ were almost the same [18]. There are also similarities between the ionic products of STX and NEO, indicating parallel dissociation mechanisms. Moreover, many ions are not the result of a simple 16 Da mass shift due to the additional 
Table 1. The chemical structures of several important product ions from STX and NEO.

\begin{tabular}{|c|c|c|c|c|c|}
\hline Ion & Structure & Ion & Structure & Ion & Structure \\
\hline 298 & & 240 & & 195 & \\
\hline 283 & & 238 & & 192 & \\
\hline 281 & & 237 & & 180 & \\
\hline 280 & & 225 & & 178 & \\
\hline 266 & & 222 & & 177 & \\
\hline 263 & & 220 & & 164 & \\
\hline 258 & & 207 & & 162 & \\
\hline 255 & & 203 & & 138 & \\
\hline 241 & & 197 & & 137 & \\
\hline
\end{tabular}


Table 2. The nominal, measured and theoretical masses, mass measurement errors (ppm), product ion identities, and resolving powers for product ions observed in the internal calibration QqTOF CID and IRMPD FT-ICR MS experiments for STX and NEO.

\begin{tabular}{|c|c|c|c|c|c|c|c|}
\hline $\begin{array}{l}\text { Calculated } \\
\text { mass }\end{array}$ & $\begin{array}{l}\text { Elemental } \\
\text { formula }\end{array}$ & $\begin{array}{c}\text { FT-ICR }(9.4 \mathrm{~T}) \\
(\mathrm{m} / \Delta \mathrm{m})\end{array}$ & $\begin{array}{l}\text { Error } \\
(\mathrm{ppm})\end{array}$ & $\begin{array}{l}\text { OqTOF (1) } \\
(\mathrm{m} / \Delta \mathrm{m})\end{array}$ & $\begin{array}{l}\text { Error } \\
(\mathrm{ppm})\end{array}$ & $\begin{array}{l}\text { OqTOF (2) } \\
(\mathrm{m} / \Delta \mathrm{m})\end{array}$ & $\begin{array}{l}\text { Error } \\
\text { (ppm) }\end{array}$ \\
\hline \multicolumn{8}{|c|}{ a. STX } \\
\hline 283.11493 & $\mathrm{C}_{10} \mathrm{H}_{15} \mathrm{~N}_{6} \mathrm{O}_{4}^{+}$ & $\begin{array}{c}283.11493 \\
(104,000)\end{array}$ & 0.0 & $\begin{array}{c}283.1241^{\mathrm{a}} \\
(6,000)\end{array}$ & 32 & $\begin{array}{c}283.1179 \\
(9,900)\end{array}$ & 11 \\
\hline 282.13091 & $\mathrm{C}_{10} \mathrm{H}_{16} \mathrm{~N}_{7} \mathrm{O}_{3}^{+}$ & $\begin{array}{l}282.13093 \\
(105,000)\end{array}$ & 0.1 & $\begin{array}{c}282.1308 \\
(9,000)\end{array}$ & -0.4 & $\begin{array}{c}282.1306 \\
(9,000)\end{array}$ & -1.1 \\
\hline 266.08838 & $\mathrm{C}_{10} \mathrm{H}_{12} \mathrm{~N}_{5} \mathrm{O}_{4}^{+}$ & $\begin{array}{c}266.08837 \\
(111,000)\end{array}$ & 0.0 & $\begin{array}{c}266.0948^{a} \\
(5,800)\end{array}$ & 24.0 & $\begin{array}{c}266.0908 \\
(6,700)\end{array}$ & 9.1 \\
\hline 265.10437 & $\mathrm{C}_{10} \mathrm{H}_{13} \mathrm{~N}_{6} \mathrm{O}_{3}{ }^{+}$ & $\begin{array}{r}265.10441 \\
(111,000)\end{array}$ & 0.2 & $\begin{array}{c}265.1045 \\
(8,700)\end{array}$ & 0.5 & $\begin{array}{c}265.1036 \\
(9,600)\end{array}$ & -2.9 \\
\hline 258.11968 & $\mathrm{C}_{9} \mathrm{H}_{16} \mathrm{~N}_{5} \mathrm{O}_{4}^{+}$ & $\begin{array}{c}258.11969 \\
(114,000)\end{array}$ & 0.0 & $\begin{array}{c}258.1203 \\
(9,500)\end{array}$ & 2.4 & $\begin{array}{c}258.1208 \\
(9,500)\end{array}$ & 4.3 \\
\hline 241.09313 & $\mathrm{C}_{9} \mathrm{H}_{13} \mathrm{~N}_{4} \mathrm{O}_{4}^{+}$ & $\begin{array}{c}241.09314 \\
(123,000)\end{array}$ & 0.0 & $\begin{array}{c}241.0987^{a} \\
(6,900)\end{array}$ & 23 & $\begin{array}{c}241.0945 \\
(6,900)\end{array}$ & 5.7 \\
\hline 240.10911 & $\mathrm{C}_{9} \mathrm{H}_{14} \mathrm{~N}_{5} \mathrm{O}_{3}^{+}$ & $\begin{array}{c}240.10912 \\
(123,000)\end{array}$ & 0.0 & $\begin{array}{c}240.1107^{\mathrm{a}} \\
(9,200)\end{array}$ & 6.6 & $\begin{array}{c}240.1090 \\
(10,300)\end{array}$ & -0.5 \\
\hline 239.12510 & $\mathrm{C}_{9} \mathrm{H}_{15} \mathrm{~N}_{6} \mathrm{O}_{2}^{+}$ & $\begin{array}{c}239.12504 \\
(124,000)\end{array}$ & -0.3 & $\begin{array}{c}239.1250 \\
(8,200)\end{array}$ & -0.4 & $\begin{array}{c}239.1253 \\
(9,200)\end{array}$ & 0.8 \\
\hline 222.09855 & $\mathrm{C}_{9} \mathrm{H}_{12} \mathrm{~N}_{5} \mathrm{O}_{2}^{+}$ & $\begin{array}{c}222.09850 \\
(133,000)\end{array}$ & -0.2 & $\begin{array}{c}222.1025^{a} \\
(6,100)\end{array}$ & 18 & $\begin{array}{c}222.1000 \\
(8,800)\end{array}$ & 6.5 \\
\hline 221.11454 & $\mathrm{C}_{9} \mathrm{H}_{13} \mathrm{~N}_{6} \mathrm{O}^{+}$ & $\begin{array}{c}221.11454 \\
(134,000)\end{array}$ & 0.0 & $\begin{array}{c}221.1147 \\
(8,800)\end{array}$ & 0.7 & $\begin{array}{c}221.1151 \\
(9,900)\end{array}$ & 2.6 \\
\hline 204.08799 & $\mathrm{C}_{9} \mathrm{H}_{10} \mathrm{~N}_{5} \mathrm{O}^{+}$ & $\begin{array}{r}204.08797 \\
(145,000)\end{array}$ & -0.1 & $\begin{array}{c}204.0882 \\
(8,500)\end{array}$ & 1.0 & $\begin{array}{c}204.0884 \\
(8,500)\end{array}$ & 2.0 \\
\hline 197.10330 & $\mathrm{C}_{8} \mathrm{H}_{13} \mathrm{~N}_{4} \mathrm{O}_{2}^{+}$ & $\begin{array}{l}197.10334 \\
(150,000)\end{array}$ & 0.2 & $\begin{array}{c}197.1027 \\
(8,300)\end{array}$ & -3.1 & $\begin{array}{c}197.1044 \\
(9,400)\end{array}$ & 5.6 \\
\hline 180.07675 & $\mathrm{C}_{8} \mathrm{H}_{10} \mathrm{~N}_{3} \mathrm{O}_{2}^{+}$ & $\begin{array}{l}180.07674 \\
(131,000)\end{array}$ & -0.1 & $\begin{array}{c}180.0846^{a} \\
(7,200)\end{array}$ & 44 & $\begin{array}{c}180.0833^{a} \\
(7,200)\end{array}$ & 36 \\
\hline 179.09274 & $\mathrm{C}_{8} \mathrm{H}_{11} \mathrm{~N}_{4} \mathrm{O}^{+}$ & $\begin{array}{l}179.09273 \\
(165,000)\end{array}$ & 0.0 & $\begin{array}{c}179.0930 \\
(7,100)\end{array}$ & 1.5 & $\begin{array}{c}179.0932 \\
(7,900)\end{array}$ & 2.6 \\
\hline 162.06619 & $\mathrm{C}_{8} \mathrm{H}_{8} \mathrm{~N}_{3} \mathrm{O}^{+}$ & $\begin{array}{l}162.06622 \\
(182,000)\end{array}$ & 0.2 & $\begin{array}{c}162.0690^{\mathrm{b}} \\
(5,700)\end{array}$ & 17 & $\begin{array}{c}162.0702^{\mathrm{b}} \\
(6,200)\end{array}$ & 25 \\
\hline 137.08217 & $\mathrm{C}_{6} \mathrm{H}_{9} \mathrm{~N}_{4}^{+}$ & $\begin{array}{l}137.08222 \\
(216,000)\end{array}$ & 0.3 & $\begin{array}{c}137.0796^{b} \\
(6,200)\end{array}$ & -19 & $\begin{array}{c}137.0785^{\mathrm{b}} \\
(5,700)\end{array}$ & -27 \\
\hline 137.07094 & $\mathrm{C}_{7} \mathrm{H}_{9} \mathrm{~N}_{2} \mathrm{O}^{+}$ & $\begin{array}{l}137.07101 \\
(216,000)\end{array}$ & 0.5 & $\begin{array}{c}137.0796^{\mathrm{b}} \\
(6,200)\end{array}$ & 63 & $\begin{array}{c}137.0785^{\mathrm{b}} \\
(5,700)\end{array}$ & 55 \\
\hline \multicolumn{8}{|c|}{ b. NEO } \\
\hline 298.12583 & $\mathrm{C}_{10} \mathrm{H}_{16} \mathrm{~N}_{7} \mathrm{O}_{4}^{+}$ & $\begin{array}{c}298.12573 \\
(99,000)\end{array}$ & -0.3 & $\begin{array}{c}298.1257 \\
(8,400)\end{array}$ & -0.4 & $\begin{array}{c}298.1242 \\
(9,200)\end{array}$ & -5.5 \\
\hline 281.09928 & $\mathrm{C}_{10} \mathrm{H}_{13} \mathrm{~N}_{6} \mathrm{O}_{4}^{+}$ & $\begin{array}{l}281.09920 \\
(105,000)\end{array}$ & -0.3 & $\begin{array}{c}281.0986^{a} \\
(8,100)\end{array}$ & -2 & $\begin{array}{c}281.0995 \\
(12,800)\end{array}$ & 0.8 \\
\hline 280.11526 & $\mathrm{C}_{10} \mathrm{H}_{14} \mathrm{~N}_{7} \mathrm{O}_{3}^{+}$ & $\begin{array}{c}280.11502 \\
(105,000)\end{array}$ & -0.9 & $\begin{array}{c}280.1157 \\
(8,100)\end{array}$ & 1.6 & $\begin{array}{r}280.1161 \\
(14,900)\end{array}$ & 3.0 \\
\hline 263.08872 & $\mathrm{C}_{10} \mathrm{H}_{11} \mathrm{~N}_{6} \mathrm{O}_{3}{ }^{+}$ & $\begin{array}{c}263.08866 \\
(112,000)\end{array}$ & -0.2 & $\begin{array}{c}263.0884 \\
(9,600)\end{array}$ & -1.2 & $\begin{array}{c}263.0876 \\
(8,700)\end{array}$ & -6.1 \\
\hline 255.12002 & $\mathrm{C}_{9} \mathrm{H}_{15} \mathrm{~N}_{6} \mathrm{O}_{3}^{+}$ & $\begin{array}{c}255.11987 \\
(116,000)\end{array}$ & -0.6 & $\begin{array}{c}255.1189 \\
(9,500)\end{array}$ & -4.4 & $\begin{array}{c}255.1189 \\
(8,700)\end{array}$ & -4.4 \\
\hline 238.09347 & $\mathrm{C}_{9} \mathrm{H}_{12} \mathrm{~N}_{5} \mathrm{O}_{3}^{+}$ & $\begin{array}{c}238.09342 \\
(124,000)\end{array}$ & -0.2 & $\begin{array}{c}238.1159^{a, b} \\
(8,300)\end{array}$ & 94 & $\begin{array}{c}238.1165^{\mathrm{b}} \\
(10,000)\end{array}$ & 97 \\
\hline 238.11728 & $\mathrm{C}_{9} \mathrm{H}_{14} \mathrm{~N}_{6} \mathrm{O}_{2}^{+}$ & $\begin{array}{l}238.11710 \\
(124,000)\end{array}$ & -0.7 & $\begin{array}{c}238.1159^{a, b} \\
(8,300)\end{array}$ & -5.8 & $\begin{array}{c}238.1165^{\mathrm{b}} \\
(10,000)\end{array}$ & -3.3 \\
\hline 237.10945 & $\mathrm{C}_{9} \mathrm{H}_{13} \mathrm{~N}_{6} \mathrm{O}_{2}^{+}$ & $\begin{array}{c}237.10942 \\
(125,000)\end{array}$ & -0.1 & $\begin{array}{c}237.1092 \\
(8,200)\end{array}$ & -1.1 & $\begin{array}{c}237.1092 \\
(10,100)\end{array}$ & -1.1 \\
\hline 225.10945 & $\mathrm{C}_{8} \mathrm{H}_{13} \mathrm{~N}_{6} \mathrm{O}_{2}^{+}$ & $\begin{array}{c}225.10941 \\
(131,000)\end{array}$ & -0.2 & $\begin{array}{c}225.1089 \\
(8,000)\end{array}$ & -2.4 & $\begin{array}{c}225.1096 \\
(8,900)\end{array}$ & 0.7 \\
\hline 220.10671 & $\mathrm{C}_{9} \mathrm{H}_{12} \mathrm{~N}_{6} \mathrm{O}^{+}$. & $\begin{array}{c}220.10667 \\
(134,000)\end{array}$ & -0.2 & $\begin{array}{c}220.0974^{a} \\
(4,600)\end{array}$ & -42 & $\begin{array}{c}220.0967^{b} \\
(4,600)\end{array}$ & -46 \\
\hline 220.08290 & $\mathrm{C}_{9} \mathrm{H}_{10} \mathrm{~N}_{5} \mathrm{O}_{2}^{+}$ & $\begin{array}{c}220.08285 \\
(134,000)\end{array}$ & -0.2 & $\begin{array}{c}220.0974^{a} \\
(4,600)\end{array}$ & 66 & $\begin{array}{c}220.0967^{b} \\
(4,600)\end{array}$ & 63 \\
\hline 207.09889 & $\mathrm{C}_{8} \mathrm{H}_{11} \mathrm{~N}_{6} \mathrm{O}^{+}$ & $\begin{array}{c}207.09888 \\
(143,000)\end{array}$ & 0.0 & $\begin{array}{c}207.0993 \\
(8,500)\end{array}$ & 2.0 & $\begin{array}{c}207.0989 \\
(8,500)\end{array}$ & 0.1 \\
\hline
\end{tabular}


Table 2. Continued

\begin{tabular}{|c|c|c|c|c|c|c|c|}
\hline $\begin{array}{l}\text { Calculated } \\
\text { mass }\end{array}$ & $\begin{array}{l}\text { Elemental } \\
\text { formula }\end{array}$ & $\begin{array}{c}\text { FT-ICR }(9.4 \mathrm{~T}) \\
(\mathrm{m} / \Delta \mathrm{m})\end{array}$ & $\begin{array}{l}\text { Error } \\
\text { (ppm) }\end{array}$ & $\begin{array}{c}\text { QqTOF (1) } \\
(\mathrm{m} / \Delta \mathrm{m})\end{array}$ & $\begin{array}{l}\text { Error } \\
\text { (ppm) }\end{array}$ & $\begin{array}{l}\text { QqTOF (2) } \\
(\mathrm{m} / \Delta \mathrm{m})\end{array}$ & $\begin{array}{l}\text { Error } \\
\text { (ppm) }\end{array}$ \\
\hline 203.05635 & $\mathrm{C}_{9} \mathrm{H}_{7} \mathrm{~N}_{4} \mathrm{O}_{2}^{+}$ & $\begin{array}{c}203.05629 \\
(145,000)\end{array}$ & -0.3 & $\begin{array}{c}203.0793^{b} \\
(6,900)\end{array}$ & 113 & $\begin{array}{c}203.0794^{b} \\
(6,900)\end{array}$ & 114 \\
\hline 203.08016 & $\mathrm{C}_{9} \mathrm{H}_{9} \mathrm{~N}_{5} \mathrm{O}^{+}$ & $\begin{array}{c}203.08013 \\
(145,000)\end{array}$ & -0.2 & $\begin{array}{c}203.0793^{b} \\
(6,900)\end{array}$ & -4.2 & $\begin{array}{c}203.0794 \\
(6,900)\end{array}$ & -3.7 \\
\hline 195.08765 & $\mathrm{C}_{8} \mathrm{H}_{11} \mathrm{~N}_{4} \mathrm{O}_{2}^{+}$ & $\begin{array}{l}195.08762 \\
(151,000)\end{array}$ & -0.2 & $\begin{array}{c}195.0860 \\
(7,500)\end{array}$ & -8.5 & $\begin{array}{c}195.0865 \\
(6,900)\end{array}$ & -5.9 \\
\hline 192.11180 & $\mathrm{C}_{8} \mathrm{H}_{12} \mathrm{~N}_{6}^{+}$. & $\begin{array}{l}192.11176 \\
(123,000)\end{array}$ & -0.2 & $\begin{array}{c}192.1097^{b} \\
(6,200)\end{array}$ & -11 & $\begin{array}{c}192.1060^{\mathrm{b}} \\
(5,700)\end{array}$ & -30 \\
\hline 192.08799 & $\mathrm{C}_{8} \mathrm{H}_{10} \mathrm{~N}_{5} \mathrm{O}^{+}$ & $\begin{array}{c}192.08791 \\
(153,000)\end{array}$ & -0.4 & $\begin{array}{c}192.0809^{b} \\
(8,200)\end{array}$ & -36 & $\begin{array}{c}192.0804^{b} \\
(8,000)\end{array}$ & -40 \\
\hline 178.06110 & $\mathrm{C}_{8} \mathrm{H}_{8} \mathrm{~N}_{3} \mathrm{O}_{2}^{+}$ & $\begin{array}{l}178.06107 \\
(166,000)\end{array}$ & -0.2 & $\begin{array}{c}178.0828^{a, b} \\
(5,900)\end{array}$ & 122 & $\begin{array}{c}178.0815^{b} \\
(5,500)\end{array}$ & 115 \\
\hline 178.07234 & $\mathrm{C}_{7} \mathrm{H}_{8} \mathrm{~N}_{5} \mathrm{O}^{+}$ & $\begin{array}{l}178.07233 \\
(166,000)\end{array}$ & 0.0 & $\begin{array}{c}178.0828^{a, b} \\
(5,900)\end{array}$ & 59 & $\begin{array}{c}178.0815^{\mathrm{b}} \\
(5,500)\end{array}$ & 52 \\
\hline 178.08491 & $\mathrm{C}_{8} \mathrm{H}_{10} \mathrm{~N}_{4} \mathrm{O}^{+}$ & $\begin{array}{l}178.08489 \\
(166,000)\end{array}$ & -0.1 & $\begin{array}{c}178.0828^{a, b} \\
(5,900)\end{array}$ & -12 & $\begin{array}{c}178.0815^{\mathrm{b}} \\
(5,500)\end{array}$ & -19 \\
\hline 177.07709 & $\mathrm{C}_{8} \mathrm{H}_{9} \mathrm{~N}_{4} \mathrm{O}^{+}$ & $\begin{array}{l}177.07708 \\
(167,000)\end{array}$ & 0.0 & $\begin{array}{c}177.0785 \\
(7,100)\end{array}$ & 8.0 & $\begin{array}{c}177.0779 \\
(7,900)\end{array}$ & 2.3 \\
\hline 138.06619 & $\mathrm{C}_{6} \mathrm{H}_{8} \mathrm{~N}_{3} \mathrm{O}^{+}$ & $\begin{array}{l}138.06619 \\
(214,000)\end{array}$ & 0.0 & $\begin{array}{c}138.0663 \\
(6,300)\end{array}$ & 0.8 & $\begin{array}{c}138.0669 \\
(7,000)\end{array}$ & 5.2 \\
\hline
\end{tabular}

The QqTOF (1) column lists the experiments with semi-internal calibration and simultaneous activation of the $\mathrm{MH}^{+}$and glutathione ions. QqTOF (2) summarizes the measurements for rapid 'back and forth' switching between $\mathrm{MH}^{+}$and glutathione and monoisotopic activation.

${ }^{a} \mathrm{M}+1$ interference.

${ }^{\mathrm{b}}$ Overlapping fragment ion species.

$-\mathrm{OH}$ group of $\mathrm{NEO}$ at $\mathrm{N}-1$. Although we are not concerned here with mechanistic details (the interested reader is referred to reference [18] for a comprehensive description of the gas-phase dissociation behavior of the PSPs), we have included a few interesting new discoveries related to the gas-phase ion chemistry of STX and NEO that we made while comparing FT-ICR and QqTOF performance (vide infra). The experiments described here focus on the range $137<m / z<300 / 316\left(\mathrm{MH}^{+}\right.$, STX/NEO), because it includes many abundant ionic products. Table 1 summarizes most of the chemical structures of the observed STX and NEO product ions employed in this study.

\section{General Considerations for Mass Accuracy Comparisons}

The experimental resolving powers shown in this study are expressed as full width at half maximum values, $m / \Delta m$ (FWHM); the theoretical resolving powers needed to distinguish between two isobaric interferences are calculated simply from the difference between the masses of the two species, $m / \Delta m_{\delta}$. The accurate mass numbers discussed in this paper for the QqTOF measurements are peak centroid data, whereas the FT-ICR numbers are the maximum of a best-fit parabola drawn through the three highest-magnitude data points.
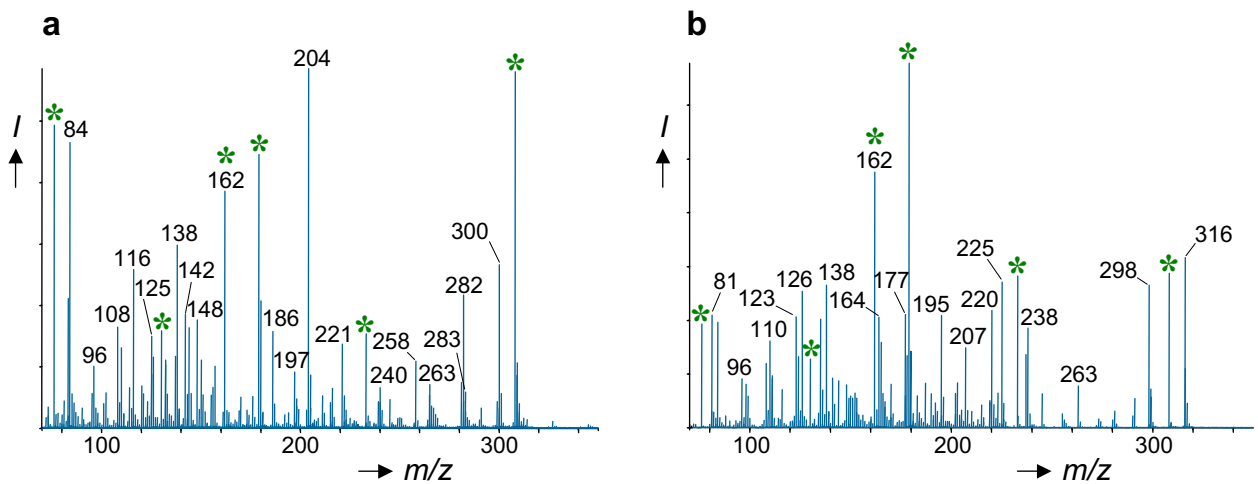

Figure 1. The Quadrupole time-of-flight CID mass spectra of STX and NEO after collisional activation of the $\mathrm{MH}^{+}$ions $(\mathrm{m} / \mathrm{z} 300$ and 316, respectively). (Note that the peaks are labeled only by their nominal masses; the accurate masses are listed in Table $2 \mathrm{a}$ and $\mathrm{b}$. Mass calibrant ions are marked with an asterisk). 
The QqTOF experiments yielded low-energy collision-induced dissociation (CID) spectra for STX and NEO (Figure 1). All spectra were then compared with results from experiments with a 9.4 tesla FT-ICR instrument. The FT-ICR MS/MS experiments employed infrared multiphoton dissociation (IRMPD) for generating the product ions. As IRMPD is rather non-selective, all trapped ions are excited simultaneously. The resulting IRMPD spectra of the PSP toxins exhibit strong similarity to QqTOF CID spectra, providing for numerous direct comparisons.

\section{Comparison of QqTOF and FT-ICR}

QqTOF experiments were initially conducted with semi-internal calibration. Our original plan to implement a true internal calibration procedure similar to the FT-ICR experiments, by activating the PSP toxin and the internal standard at the same time in $\mathrm{q}_{2}$, giving CID spectra with in situ reference ions, proved to be difficult because the optimum collision energies for the PSPs and glutathione were quite different. To circumvent this problem, we sequentially infused the analyte and the calibrant solutions and adjusted the collision energy in between, without ever stopping data acquisition. This procedure was very close to internal mass calibration because all experimental parameters (except the collision energy) were identical and the resulting spectra contained product ions of the analyte and the calibrant (Scheme 2). More importantly, the CID spectra exhibited all of the important characteristic features, in particular the excitation of multiple species in $\mathrm{q}_{2}$ (the single-ion monitoring [SIM] isolation window of $\mathrm{Q}_{1}$ was widened to allow passage of $\mathrm{MH}^{+}$ions of PSP and glutathione [STX, range, $300<\mathrm{m} / \mathrm{z}<308$; NEO, $308<$ $m / z<316])$. All spectra were then compared with results from IRMPD FT-ICR experiments, for which true internal calibration was implemented by use of alternated dual electrospray of PSP and calibrant ions ([Glu $\left.{ }^{1}\right]$-fibrinopeptide) into an external octopole trap, followed by injection into the ICR cell for additional SWIFT isolation prior to IRMPD (see the Experimental section).

The resulting mass accuracies and resolving powers from QqTOF and FT-ICR measurements are summarized in Table 2a and b (note that the QqTOF results from these experiments are listed in the QqTOF (1) column). In IRMPD FT-ICR, accurate masses with measurement uncertainties $<1 \mathrm{ppm}$ were achieved for all product ions, allowing unambiguous assignment of only one possible elemental formula to every fragment ion. Many of the same product ions were also observed with excellent mass accuracy in the QqTOF spectra, often with measurement uncertainties $<5 \mathrm{ppm}$. A significant number of the QqTOF MS/MS products, however, exhibited unusually large deviations from the expected $\mathrm{m} / \mathrm{z}$ values, with some measurement errors higher than $100 \mathrm{ppm}$. A closer look at those peaks and
Table 3. The theoretical resolving power, $m / \Delta m_{\delta}$, required to separate several important interfered species in the MS/MS spectra of STX and NEO

\begin{tabular}{|c|c|c|c|}
\hline $\begin{array}{l}\text { Nominal } \\
\text { mass }\end{array}$ & $\begin{array}{c}\text { Calculated } \\
\text { masses }\end{array}$ & Formulae & $\begin{array}{c}m / \Delta m \delta \\
\text { required }\end{array}$ \\
\hline \multicolumn{4}{|c|}{ NEO } \\
\hline 281 & $\begin{array}{l}281.09928 \\
281.11862\end{array}$ & $\begin{array}{l}\mathrm{C}_{10} \mathrm{H}_{13} \mathrm{~N}_{6} \mathrm{O}_{4}{ }^{+} \\
{ }^{12} \mathrm{C}_{9}{ }^{13} \mathrm{C}_{1} \mathrm{H}_{14} \mathrm{~N}_{7} \mathrm{O}_{3}{ }^{+}\end{array}$ & 14,529 \\
\hline 238 & $\begin{array}{l}238.09347 \\
238.11281 \\
238.11728\end{array}$ & $\begin{array}{l}\mathrm{C}_{9} \mathrm{H}_{12} \mathrm{~N}_{5} \mathrm{O}_{3}{ }^{+} \\
{ }^{12} \mathrm{C}_{8}{ }^{13} \mathrm{C}_{1} \mathrm{H}_{13} \mathrm{~N}_{6} \mathrm{O}_{2}{ }^{+} \\
\mathrm{C}_{9} \mathrm{H}_{14} \mathrm{~N}_{6} \mathrm{O}_{2}^{+}\end{array}$ & $\begin{array}{l}12,307 \\
53,243\end{array}$ \\
\hline 220 & $\begin{array}{l}220.08920 \\
220.10671\end{array}$ & $\begin{array}{l}\mathrm{C}_{9} \mathrm{H}_{10} \mathrm{~N}_{5} \mathrm{O}_{2}^{+} \\
\mathrm{C}_{9} \mathrm{H}_{12} \mathrm{~N}_{6} \mathrm{O}^{+}\end{array}$ & 12,564 \\
\hline 203 & $\begin{array}{l}203.05635 \\
203.08016\end{array}$ & $\begin{array}{l}\mathrm{C}_{9} \mathrm{H}_{7} \mathrm{~N}_{4} \mathrm{O}_{2}^{+} \\
\mathrm{C}_{9} \mathrm{H}_{9} \mathrm{~N}_{5} \mathrm{O}^{+}\end{array}$ & 8,526 \\
\hline 192 & $\begin{array}{l}192.08799 \\
192.11180\end{array}$ & $\begin{array}{l}\mathrm{C}_{8} \mathrm{H}_{10} \mathrm{~N}_{5} \mathrm{O}^{+} \\
\mathrm{C}_{8} \mathrm{H}_{12} \mathrm{~N}_{6}{ }^{+}\end{array}$ & 8,065 \\
\hline 178 & $\begin{array}{l}178.06110 \\
178.07234 \\
178.08044 \\
178.08491\end{array}$ & $\begin{array}{l}\mathrm{C}_{8} \mathrm{H}_{8} \mathrm{~N}_{3} \mathrm{O}_{2}^{+} \\
\mathrm{C}_{7} \mathrm{H}_{8} \mathrm{~N}_{5} \mathrm{O}^{+} \\
{ }^{12} \mathrm{C}_{7}{ }^{13} \mathrm{C}_{1} \mathrm{H}_{9} \mathrm{~N}_{4} \mathrm{O}^{+} \\
\mathrm{C}_{8} \mathrm{H}_{10} \mathrm{~N}_{4} \mathrm{O}^{+}\end{array}$ & $\begin{array}{l}21,975 \\
39,821\end{array}$ \\
\hline \multicolumn{4}{|c|}{ STX } \\
\hline 283 & $\begin{array}{l}283.11493 \\
283.13427\end{array}$ & $\begin{array}{l}\mathrm{C}_{10} \mathrm{H}_{15} \mathrm{~N}_{6} \mathrm{O}_{4}{ }^{+} \\
{ }^{12} \mathrm{C}_{9}{ }^{13} \mathrm{C}_{1} \mathrm{H}_{16} \mathrm{~N}_{7} \mathrm{O}_{3}{ }^{+}\end{array}$ & 14,633 \\
\hline 241 & $\begin{array}{l}241.09313 \\
241.11247\end{array}$ & $\begin{array}{l}\mathrm{C}_{9} \mathrm{H}_{13} \mathrm{~N}_{4} \mathrm{O}_{4}^{+} \\
{ }^{12} \mathrm{C}_{8}{ }^{13} \mathrm{C}_{1} \mathrm{H}_{14} \mathrm{~N}_{5} \mathrm{O}_{3}{ }^{+}\end{array}$ & 12,461 \\
\hline 222 & $\begin{array}{l}222.09855 \\
222.11789\end{array}$ & $\begin{array}{l}\mathrm{C}_{9} \mathrm{H}_{12} \mathrm{~N}_{5} \mathrm{O}_{2}{ }^{+} \\
{ }^{12} \mathrm{C}_{8}{ }^{13} \mathrm{C}_{1} \mathrm{H}_{13} \mathrm{~N}_{6} \mathrm{O}^{+}\end{array}$ & 11,478 \\
\hline 180 & $\begin{array}{l}180.07675 \\
180.09609\end{array}$ & $\begin{array}{l}\mathrm{C}_{8} \mathrm{H}_{10} \mathrm{~N}_{3} \mathrm{O}_{2}{ }^{+} \\
{ }^{12} \mathrm{C}_{7}{ }^{13} \mathrm{C}_{1} \mathrm{H}_{11} \mathrm{~N}_{4} \mathrm{O}^{+}\end{array}$ & 9,308 \\
\hline 137 & $\begin{array}{l}137.07094 \\
137.08217\end{array}$ & $\begin{array}{l}\mathrm{C}_{7} \mathrm{H}_{9} \mathrm{~N}_{2} \mathrm{O}^{+} \\
\mathrm{C}_{6} \mathrm{H}_{9} \mathrm{~N}_{4}{ }^{+}\end{array}$ & 12,196 \\
\hline
\end{tabular}

a direct comparison with the corresponding FT-ICR results revealed multiple species and overlapping peaks from isobaric interferences as the source of the mass errors.

As noted above, the interferences in the QqTOF spectra can be grouped into the three categories: isobaric interferences from multiple species formed via different fragmentation pathways, isobaric ${ }^{13} \mathrm{C}$ containing $(\mathrm{M}+1)$ species, and isobaric analyte/calibrant product ions. We were able to minimize the interferences from the latter by carefully choosing a suitable reference substance (glutathione), with a molecular weight between those of the two PSP toxins. Two product ions from the calibrant at $\mathrm{m} / \mathrm{z} 162$ and 179 came close to STX product ions (Table 2). In theory, a resolving power of only $m / \Delta m_{\delta} \approx 3500$ is required to separate them and the QqTOF instrument should be able to easily resolve the isobaric species. However, only the $\mathrm{m} / \mathrm{z} 179$ ions were identified by QqTOF, whereas the $m / z 162$ peak exhibited a surprisingly large measurement uncertainty (Table 2). A possible explana- 


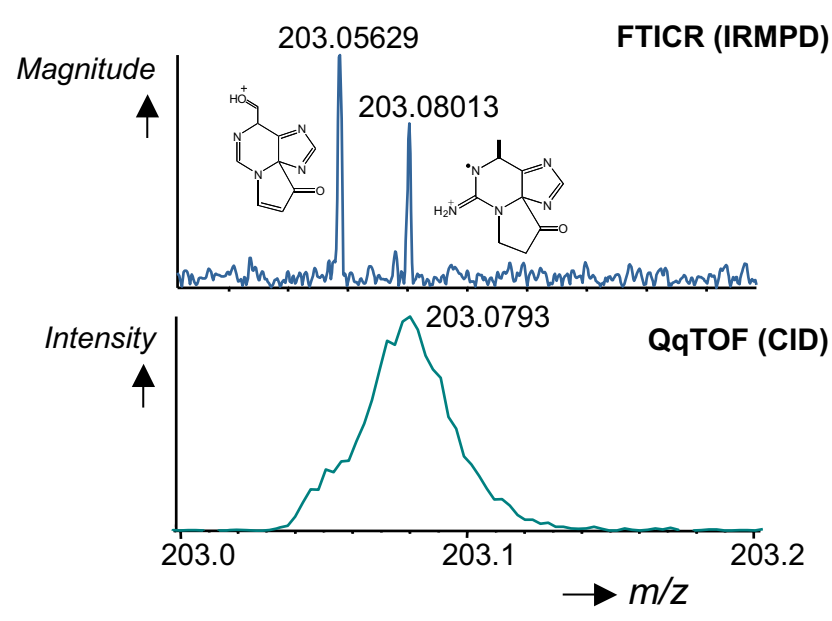

Figure 2. Two isobaric NEO product ions at $\mathrm{m} / \mathrm{z}$ 203. (Top) IRMPD FT-ICR mass scale expansion of the isobaric region; (bottom) corresponding QqTOF spectrum.

tion is the presence of a third interfering species at $\mathrm{m} / \mathrm{z}$ 162 (expected elemental formula $\mathrm{C}_{7} \mathrm{H}_{8} \mathrm{~N}_{5}{ }^{+}$), whose analog was observed in the NEO spectrum $(\mathrm{m} / \mathrm{z} 178$, $\mathrm{C}_{7} \mathrm{H}_{8} \mathrm{~N}_{5} \mathrm{O}^{+}$). Unfortunately, we could not confirm the identity of the $\mathrm{C}_{7} \mathrm{H}_{8} \mathrm{~N}_{5}{ }^{+}$ion from the FT-ICR spectrum. The most interesting interferences and the theoretical resolving power needed to separate the isobars are summarized in Table 3.

The first group of potential interferences comprises multiple isobaric species formed from the $\mathrm{MH}^{+}$ion and through subsequent dissociations; e.g., $m / z 137$ for STX and $m / z$ 220, 203, and 192 for NEO. NEO exhibits more interfering isobaric products because most originate via a radical loss of the hydroxyl group at $\mathrm{N}-1$ (vide infra). Theoretical resolving power in the range $m / \Delta m_{\delta}=8000$ to 12,500 is necessary to separate the peak pairs, out of reach of the QqTOF instrument (Table 3), which was able to provide resolution only in the range $m / \Delta m$ $(\mathrm{FWHM})=6900$ to 9900 (note that similarly insufficient $m / \Delta m$ (FWHM) values were obtained with a second QqTOF instrument from a different manufacturer; data not shown). For example, resolution of the two isobaric fragment ion species at $\mathrm{m} / \mathrm{z} 203$ of NEO (Table 1) requires a theoretical resolving power of $m / \Delta m_{\delta} \approx$ 8500. Figure 2 illustrates this deficiency compared to the unambiguous results of the corresponding IRMPD FTICR experiments. In IRMPD MS/MS, $m / \Delta m$ (FWHM) ranged from $99,000(\mathrm{~m} / \mathrm{z} 298)$ to $216,000(\mathrm{~m} / \mathrm{z} 137)$ for STX and NEO. Similarly, the species at $m / z 137$ (STX) as well as $\mathrm{m} / \mathrm{z} 220$ and 192 could not be resolved into their doublets with the QqTOF instrument (Table 3). The identities of those ions are further discussed below.

The relative contributions of two isobaric species in the TOF spectra can be estimated by mass-domain based signal deconvolution, as recently outlined by Meija and Caruso [35]. It was shown that the observed centroid mass is the weighted average of the isobar masses when the TOF peak width $\Delta m$ is larger than the mass difference of the isobars, $\Delta m \geq m_{\mathrm{A}}-m_{\mathrm{B}}$ :

$m_{\max } \cong x_{A} m_{A}+x_{B} m_{B,}$

where $m_{\max }$ is the maximum amplitude of the observed composite peak and $x_{\mathrm{A}}$ and $x_{\mathrm{B}}$ are the relative abundances of the ions of masses $m_{\mathrm{A}}$ and $m_{\mathrm{B}}$. For the example discussed above (NEO, $m / z$ 203; Figure 2), the mass difference between $\mathrm{C}_{9} \mathrm{H}_{7} \mathrm{~N}_{4} \mathrm{O}_{2}{ }^{+}(203.05635 \mathrm{u})$ and $\mathrm{C}_{9} \mathrm{H}_{9} \mathrm{~N}_{5} \mathrm{O}^{+\cdot}(203.08016 \mathrm{u})$ is $24 \mathrm{mDa}$ whereas the estimated peak width $\Delta m$ is about $26 \mathrm{mDa}$. The above equation yields relative abundances of $4 \%$ for $\mathrm{C}_{9} \mathrm{H}_{7} \mathrm{~N}_{4} \mathrm{O}_{2}{ }^{+}$and $96 \%$ for $\mathrm{C}_{9} \mathrm{H}_{9} \mathrm{~N}_{5} \mathrm{O}^{+\cdot}$. Not surprisingly, the TOF mass measurement gave accurate results for $\mathrm{C}_{9} \mathrm{H}_{9} \mathrm{~N}_{5} \mathrm{O}^{+\cdot}$ ion with an error of only $-4.2 \mathrm{ppm}$. Conversely, the elemental formula fit for $\mathrm{C}_{9} \mathrm{H}_{7} \mathrm{~N}_{4} \mathrm{O}_{2}{ }^{+}$was not very accurate (112 ppm) because the peak centroid maximum was shifted almost entirely away from this low abundant component. Of course, for convolved peaks for which both species contribute significantly, inaccurate results are obtained for both isobars. For example, the two species contributing to the isobaric signal at $m / z 220, \mathrm{C}_{9} \mathrm{H}_{10} \mathrm{~N}_{5} \mathrm{O}_{2}{ }^{+}$and $\mathrm{C}_{9} \mathrm{H}_{12} \mathrm{~N}_{6} \mathrm{O}^{+}$, are 24 $\mathrm{mDa}$ apart. The TOF peak width for a non-interfered peak at $\mathrm{m} / \mathrm{z} 220$ was estimated to be $26 \mathrm{mDa}$, so the above equation could be applied. As a result, the relative contributions of $x_{\mathrm{A}}$ and $x_{\mathrm{B}}$ were 39 and $61 \%$. Consequently, the elemental formula calculations did not yield a good fit for either species ( -42 and $66 \mathrm{ppm}$,

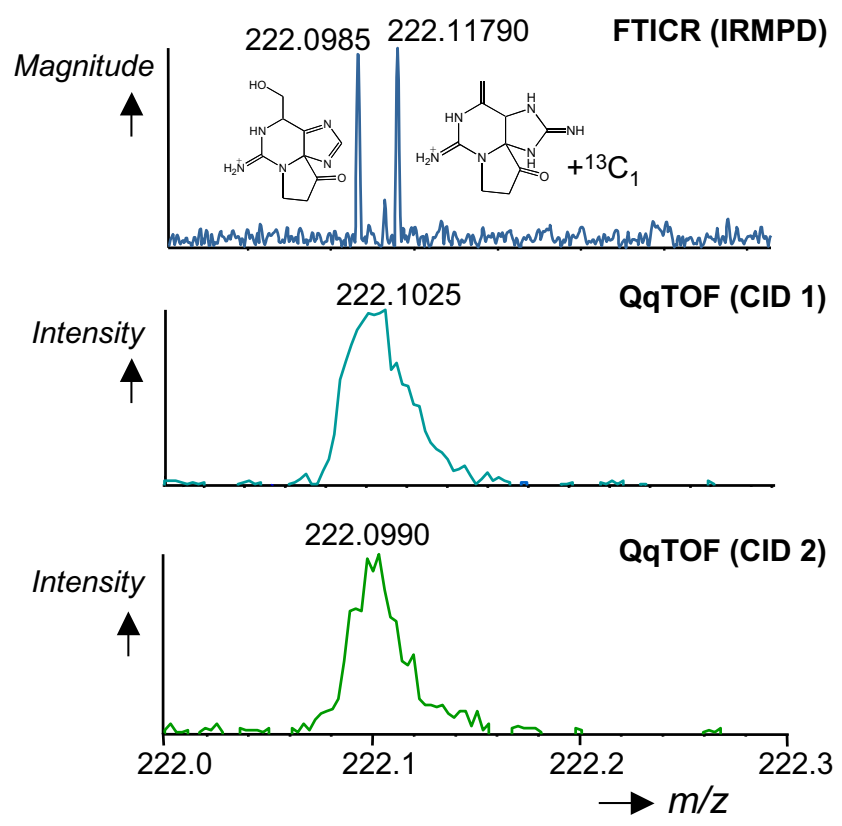

Figure 3. Isobaric ${ }^{12} \mathrm{C}_{9}$ and ${ }^{13} \mathrm{C}_{1}{ }^{12} \mathrm{C}_{8}$ species at $\mathrm{m} / \mathrm{z} 222$ in STX product ion spectra. (Top) mass scale expansion of the isobaric IRMPD FT-ICR spectrum; (middle) QqTOF spectrum after coactivation of $m / z 222$ and calibrant ions; (bottom) QqTOF spectrum after monoisotopic activation of $\mathrm{m} / \mathrm{z} 222$ ions. 
a<smiles>[R16][Z16]1CCN2C(=N)N(O)C(=C)C3NC(=N)NC132</smiles>

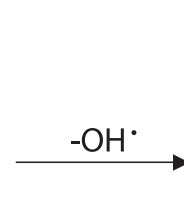<smiles>C=C1C2NC(=N)NC23CCN3C(=N)N1O</smiles>
$\mathrm{m} / \mathrm{z} 209$ (only in IRMPD)

b
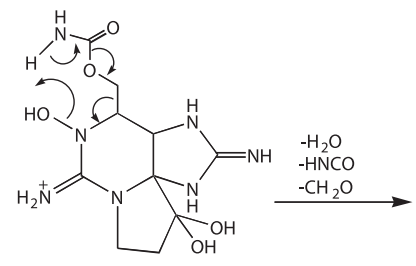

$\mathrm{m} / \mathrm{z3} 16$

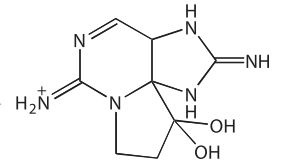

$\mathrm{m} / \mathrm{z} 225$

Scheme 3. Proposed dissociation mechanisms for several interesting fragmentation reactions in the product ion spectra of NEO. (a) Formation of $\mathrm{m} / \mathrm{z} 220$ and 192 in the spectrum of NEO; (b) formation of $\mathrm{m} / \mathrm{z} 225$ from $\mathrm{MH}^{+}$of NEO.

respectively), because the centroid mass was shifted to an intermediate value between the two peaks.

The other major interference category originates from $\mathrm{M}+1$ contributions, due to co-collisional activation of the precursor's ${ }^{13} \mathrm{C}^{12} \mathrm{C}_{\mathrm{c}-1}$ ions. As a result, multiple potential interferences occur in segments of high peak density in the MS/MS spectra. These interferences are highlighted in Table 3. To separate all ${ }^{12} \mathrm{C}_{\mathrm{c}}$ products from ${ }^{13} \mathrm{C}^{12} \mathrm{C}_{\mathrm{c}-1}$ species, even higher resolving power required, up to $m / \Delta m_{\delta}=53,000$, well beyond the capability of QqTOF instruments. Incidentally, the two interfering species were always of the general formulas $\mathrm{C}_{\mathrm{c}} \mathrm{H}_{\mathrm{x}} \mathrm{N}_{\mathrm{y}} \mathrm{O}_{\mathrm{z}}$ and ${ }^{13} \mathrm{C}_{1}{ }^{12} \mathrm{C}_{\mathrm{c}-1} \mathrm{H}_{\mathrm{x}+1} \mathrm{~N}_{\mathrm{y}+1} \mathrm{O}_{\mathrm{z}-1}$; i.e., $\Delta m$ was always $19.4 \mathrm{mDa}$. The QqTOF experimental resolving power, however, was never high enough to resolve these two species, whereas the FT-ICR instrument was always well above the required theoretical resolving power, resulting in clearly separated isobars (Figure 3 ).

The $\mathrm{M}+1$ interferences in the QqTOF spectra clearly result from a specific weakness of the applied semiinternal calibration routine, as the procedure activates unwanted ${ }^{13} \mathrm{C}^{12} \mathrm{C}_{\mathrm{c}-1}$ species at the same time. Although the isobaric fragment ion interferences obviously cannot be avoided, $\mathrm{M}+1$ interferences would not occur with monoisotopic isolation and activation of the $\mathrm{MH}^{+}$ ions, as with external calibration. In order to combine the advantages of internal calibration and monoisotopic activation and thus reduce or completely eliminate

some of these $\mathrm{M}+1$ interferences, we changed the data acquisition protocol.

\section{The Refined Approach: Monoisotopic Activation Cycles}

For our improved acquisition technique, we modified the QqTOF instrument software to allow rapid "back and forth" switching between the $\mathrm{MH}^{+}$ion of the analyte and that of the mass calibrant (see the Experimental section for details). The results of the modified procedure are summarized in Table $2 \mathrm{a}$ and $\mathrm{b}$ for comparison to the previous method. The $M+1$ interferences are clearly reduced significantly compared to the previous activation method. The improvement is particularly important for mass assignments in the STX spectra as more isotope interferences are seen compared to NEO. An example for the improved peak centroid is shown in Figure 3 for the STX fragment ion at $\mathrm{m} / \mathrm{z} 222$ $\left(\mathrm{C}_{9} \mathrm{H}_{12} \mathrm{~N}_{5} \mathrm{O}_{2}{ }^{+}\right.$, calculated mass at $m / z$ 222.0986). Note how the centroid mass shifts from $\mathrm{m} / \mathrm{z} 222.1025$ to 222.0990 by eliminating the mass shift-causing ${ }^{13} \mathrm{C}$ isotope of $\mathrm{m} / \mathrm{z} 221$. At the same time, the uncertainty of measurement improves from $18 \mathrm{ppm}$ to $6.5 \mathrm{ppm}$. By comparison, the FT-ICR spectrum clearly shows two well-separated signals for the two species present simultaneously (because they were produced by IRMPD, the relative abundances differ somewhat from the QqTOF CID MS/MS spectrum). Some product ions still exhibit small mass centroid shifts because the chosen resolution setting of $Q_{1}$ was not narrow enough and allowed a small number of ${ }^{13} \mathrm{C}^{12} \mathrm{C}_{\mathrm{c}-1}$ ions to pass through into $\mathrm{q}_{2}$ before TOF analysis. These minor interferences then had an effect on low abundance fragments such as $\mathrm{m} / \mathrm{z} 180$ in the spectrum of STX. Although the ${ }^{13} \mathrm{C}^{12} \mathrm{C}_{\mathrm{c}-1}$ contribution from highly abundant $\mathrm{m} / \mathrm{z} 179$ was almost completely eliminated in the $\mathrm{Q}_{1}$ isolation step, the very small residual signal still interfered with $\mathrm{m} / \mathrm{z} 180$ (Table 2), with the result that the mass measurement error dropped only from $44 \mathrm{ppm}$ to 36 ppm. Such "cross-talk" could be completely eliminated by increased $Q_{1}$ resolution, at the cost of reduction in signal. Of course, the inherent isobaric interferences (e.g., $m / z$ 238, 220, 203, 192, 178, and 137) from different characteristic fragment ion species, as described in the previous section, are not eliminated by the modified procedure and represent the ultimate limitation for applying a QqTOF instrument to the analysis of PSP toxin MS/MS spectra.

Interestingly, the observation of peaks yielding poor mass accuracy of the QqTOF system offers an unexpected but convenient way for detecting the presence of multiple species in a composite peak, many of which had previously escaped our attention when investigating the triple-quadrupole CID behavior of PSPs [18]. Some of the more important examples are illustrated in the following section. 


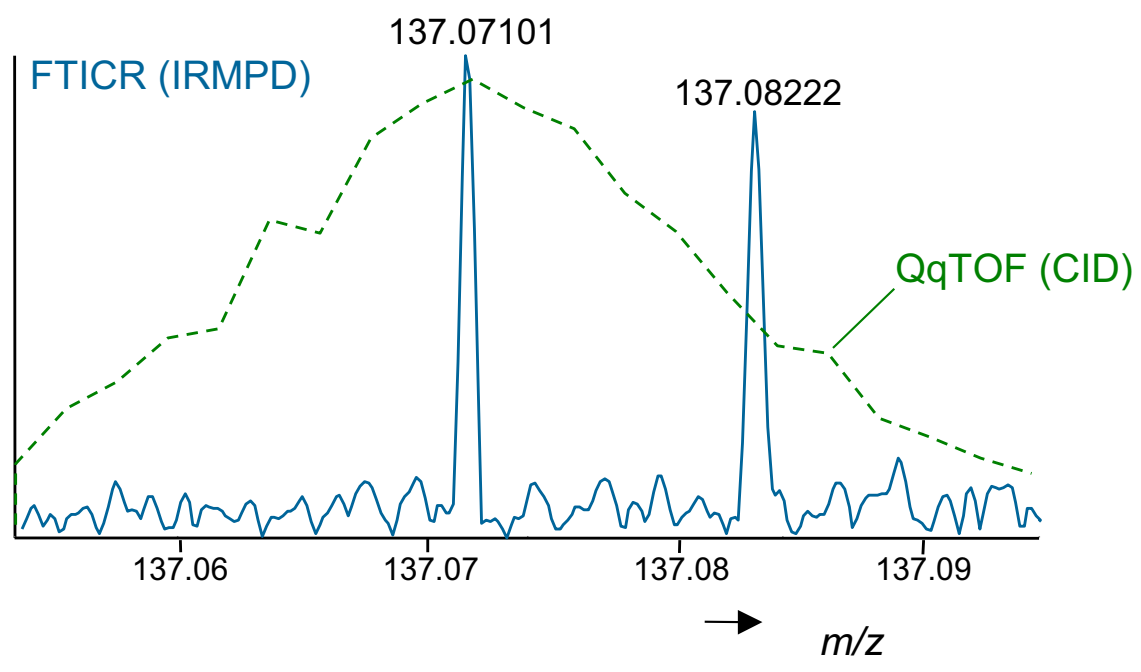

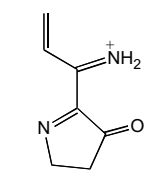

$\mathrm{C}_{7} \mathrm{H}_{9} \mathrm{~N}_{2} \mathrm{O}^{+}$

$m / z 137.07094$

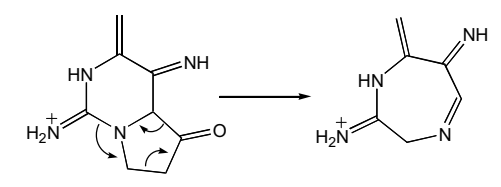

$m / z 179$

$$
\begin{aligned}
& \mathrm{C}_{6} \mathrm{H}_{9} \mathrm{~N}_{4}{ }^{+} \\
& m / z 137.08217
\end{aligned}
$$

Figure 4. Two isobaric species differing by $\mathrm{CO}$ versus $\mathrm{N}_{2}(0.01123 \mathrm{Da})$ at $\mathrm{m} / \mathrm{z} 137$ in product ion spectra of STX. The measured masses are given for the IRMPD FT-ICR experiment. The spectrum obtained under QqTOF CID conditions is shown as a dashed line. The chemical structures of the two product ions as well as their calculated exact masses are shown below the spectrum.

\section{A Few Interesting Observations in the QqTOF CID Spectra of STX and NEO}

We previously presented a comprehensive dissociation scheme for STX and NEO, observed under low-energy CID conditions. That scheme described the fragmentation behavior in the collision cell of a triple-quadrupole instrument. The identification of the product ions in the CID spectra was supported by linked ion-trap $\mathrm{MS}^{n}$ data for the tentative dissociation schemes, followed by subsequent high-resolution FT-ICR experiments for accurate mass data [18]. Because the accurate mass measurements were only used for confirmation of tentatively identified product ions, some of the isobaric ions discussed in the present paper remained undetected at the time and were not structurally investigated previously. The mechanisms of formation are interesting enough, however, to report them here briefly as an extension to the previous study.

The most interesting observation is the formation of several radical ion species. NEO exhibits a number of radical hydroxyl losses from $\mathrm{N}-1$, which were, of course, not observed in the corresponding STX spectra, because STX does not possess a $-\mathrm{OH}$ function at $\mathrm{N}-1$. Examples in the NEO CID spectra are $\mathrm{m} / \mathrm{z} 220$ and 192, which are related ions (Scheme 3a). As mentioned above, we discovered these new species because several peaks in the QqTOF spectra display unusually large mass measurement errors, pointing to multiple species under the peak.
The elemental compositions are given in Tables 2 and 3. Because there were no products ions at $\mathrm{m} / \mathrm{z} 219$ and 191 in the MS/MS spectrum of NEO, M +1 interferences are ruled out. Instead, as confirmed by FT-ICR, the additional species are formed by loss of $\cdot \mathrm{OH}$ and loss of $\mathrm{CO}$ from $\mathrm{m} / \mathrm{z}$ 237 via the $\mathrm{m} / \mathrm{z} 220$ and 209 intermediates (Scheme 3a). A similar mechanism was proposed for $m / z 203$ [18]. Another interesting NEO-specific fragment was observed at $\mathrm{m} / \mathrm{z} 225$, the proposed mechanism of formation of which is illustrated in Scheme $\mathbf{3 b}$. This intriguing mechanism involves a concerted loss of three small neutral molecules involving the hydroxyl group at $\mathrm{N}-1$ and the carbamate function at C-6.

The next example illustrates two interfering isobars at $m / z 137$ in the product ion spectrum of STX that remained undetected in our previous study. The first species is the result of a $\mathrm{HN}=\mathrm{C}=\mathrm{NH}$ loss from $\mathrm{m} / \mathrm{z} 179$ after opening of the imidazoline ring $\left(\mathrm{C}_{6} \mathrm{H}_{9} \mathrm{~N}_{4}{ }^{+}\right.$; Figure 4). For the second $m / z 137$ species we now propose the structure in Figure $4\left(\mathrm{C}_{7} \mathrm{H}_{9} \mathrm{~N}_{2} \mathrm{O}^{+}\right)$, based on the FT-ICR data. The two signals are only $11.2 \mathrm{mDa}$ apart in the QqTOF CID spectrum; thus, the signal deconvolution calculation spectra described above reveals a contribution of $33 \%$ for $\mathrm{C}_{7} \mathrm{H}_{9} \mathrm{~N}_{2} \mathrm{O}^{+}$and $67 \%$ for $\mathrm{C}_{6} \mathrm{H}_{9} \mathrm{~N}_{4}{ }^{+}$, correlating well with the observed mass uncertainties (Table 2).

Finally, the isobars at $\mathrm{m} / \mathrm{z} 178$ and 238 of NEO provide perfect examples for illustrating the need for 


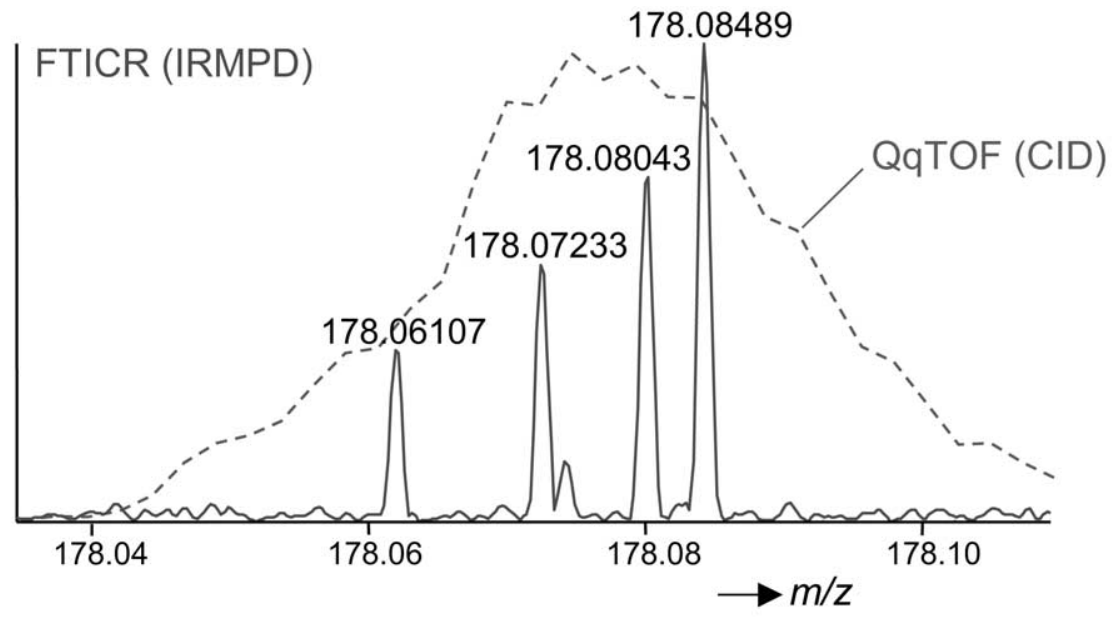

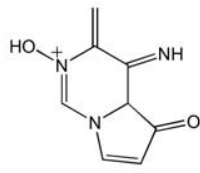

$\mathrm{C}_{8} \mathrm{H}_{8} \mathrm{~N}_{3} \mathrm{O}_{2}^{+}$ $\mathrm{m} / \mathrm{z} 178.06110$

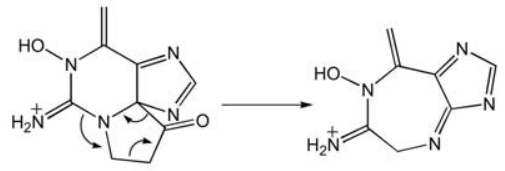

$m / z 220$

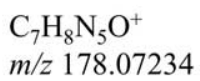

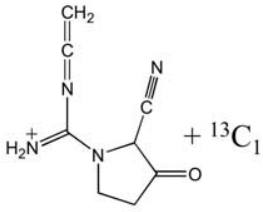

${ }^{12} \mathrm{C}_{7}{ }^{13} \mathrm{C}_{1} \mathrm{H}_{9} \mathrm{~N}_{4} \mathrm{O}^{+}$

$\mathrm{m} / \mathrm{z} 178.08044$

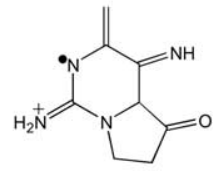

$\mathrm{C}_{8} \mathrm{H}_{10} \mathrm{~N}_{4} \mathrm{O}^{+*}$ $\mathrm{m} / \mathrm{z} 178.08491$

Figure 5. Four isobaric species at $\mathrm{m} / \mathrm{z} 178$ in the IRMPD product ion spectrum of NEO. The ion structures as well as their calculated exact masses are shown below the spectrum. For comparison, the QqTOF CID spectrum is given as a dashed line.

FT-ICR over QqTOF in structural studies of the type described here (Figures 5 and 6). Initially, we suspected an $\mathrm{M}+1$ interference from $\mathrm{m} / \mathrm{z} 177\left({ }^{12} \mathrm{C}_{7}{ }^{13} \mathrm{C}_{1} \mathrm{H}_{9} \mathrm{~N}_{4} \mathrm{O}^{+}\right)$ to contribute to the $\mathrm{C}_{8} \mathrm{H}_{10} \mathrm{~N}_{4} \mathrm{O}^{+}$signal at $\mathrm{m} / \mathrm{z} 178$, thus shifting the mass centroid away from the true mass. The monoisotopic selection and activation of $\mathrm{MH}^{+}$(vide supra), however, did not improve the mass accuracy (Table 2) for $\mathrm{C}_{8} \mathrm{H}_{10} \mathrm{~N}_{4} \mathrm{O}_{3}{ }^{+}$, pointing to a different or additional source of interference. A closer look at the FT-ICR spectrum revealed an interesting situation: the $\mathrm{m} / \mathrm{z} 178$ signal consists of four isobars within only 0.025 Da $\left(\mathrm{C}_{8} \mathrm{H}_{8} \mathrm{~N}_{3} \mathrm{O}_{2}{ }^{+}\right.$and $\mathrm{C}_{7} \mathrm{H}_{8} \mathrm{~N}_{5} \mathrm{O}^{+}$plus the two species mentioned above; Scheme 3); only one of them is an actual ${ }^{13} \mathrm{C}^{12} \mathrm{C}_{\mathrm{c}-1}$ interference (Scheme 3), explaining the remaining interference after monoisotopic activation. In contrast, the limited resolving power of the QqTOF instrument yielded only one coalescent signal. A similar situation was encountered for $m / z 238$ (Figure 6), where a quadruplet signal forms the composite peak. Again, the similar mass accuracies for the two mass calibration procedures in Table 2 point to at least one additional interference other than $\mathrm{M}+1$ contributing to the signal. Indeed, the FT-ICR analysis resolved the peak into four separate species at $\mathrm{m} / \mathrm{z} 238$ (Figure 6).

\section{Prospects for Automated Formulas Assignment and Other Practical Issues}

This final section summarizes the findings of the instrument comparison and puts them in a practical context.
To illustrate this, we express the overall quality of the mass measurements for PSP product ions as a "first hit" rate, namely, the fraction of correct first-choice software assignments of elemental compositions as described by Hau and coworkers [20]. For most small molecules with their usually very simple CID spectra, hit rate depends mainly on the error of the mass measurement alone, but not for the complex spectra described in this study. Here, as described in the previous sections, both high mass accuracy and high resolving power must be available simultaneously. Not surprisingly, error-free assignments were possible only with FT-ICR yielding a perfect $100 \%$ first hit rate. From the data in Table 2, the QqTOF first hit rate was only 56 and 65\% for STX and NEO products, based on internal calibration with concurrent activation of the protonated molecules of PSPs and internal calibrant. The hit rate improves to $75 \%$ for STX with alternated data acquisition with monoisotopic activation, by largely eliminating the ${ }^{13} \mathrm{C}^{12} \mathrm{C}_{\mathrm{c}-1}$ interferences in the spectrum. The QqTOF first hit rate for NEO remained at $65 \%$ with either calibration method, because the interferences in the NEO spectra originate almost exclusively from different fragment ion pathways. Interestingly, the QqTOF hit rate in our experiments approached almost $100 \%$ for both STX and NEO when only the non-interfered species were considered. That finding is important for routine QqTOF analyses, where less demanding analytical problems are more common, showing that automated formulas assignments are readily possible for pure samples in regular 


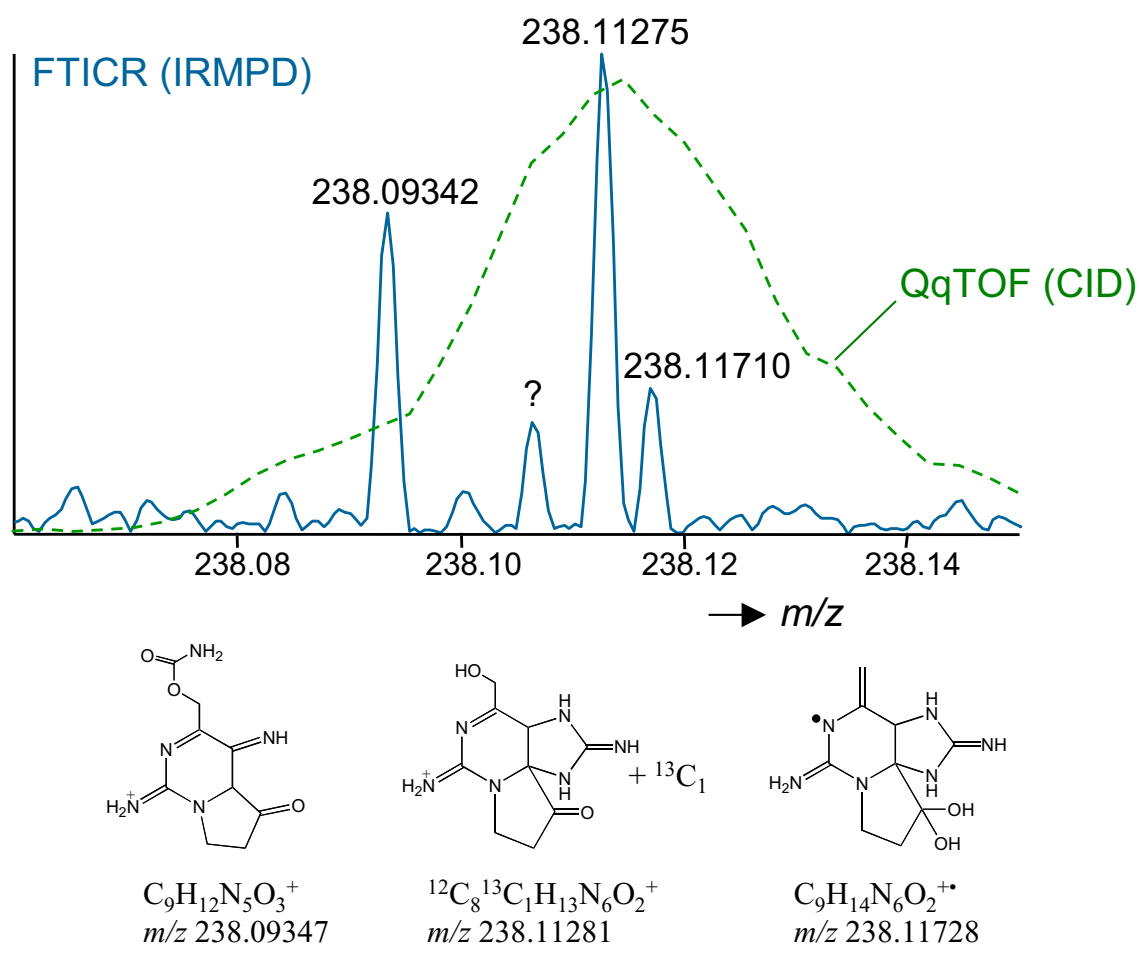

Figure 6. The segment near $\mathrm{m} / \mathrm{z} 238$ reveals at least four isobaric species in the IRMPD spectrum of NEO. The structures of three of them as well as their calculated exact masses are given below the spectrum. The dashed line illustrates the QqTOF CID spectrum for comparison.

MS mode or for interference-free MS/MS spectra. Of course, since one never knows in advance whether potential interferences are present, only high mass resolution can give sample-independent reliable mass measurement.

To clarify this issue, we have also compared the mass measurement accuracies obtained with a commercial 7 tesla FT-ICR instrument to QqTOF data. In that comparison, only external calibration was applied prior to conducting the measurements on both instruments and the mass values were taken directly from the spectra, without any further processing. The results of those experiments are summarized in Table 4. Naturally, the same isobaric interferences as discussed above occurred in the QqTOF spectra and will not be discussed further. The important point is that under experimental conditions that many routine labs would choose, the mass measurement uncertainties of FT-ICR and QqTOF are quite similar, in the absence of peak overlap. The superior resolving power of the FT-ICR $(\mathrm{m} / \Delta \mathrm{m}$ (FWHM) between 60,000 to 150,000 ), however, is still seen in this comparison, in which interfering species are completely resolved, whereas accuracies still remain low for the QqTOF data.

The primary advantage of QqTOF over FT-ICR is higher sensitivity because TOF detectors approach single-ion detection limit, whereas FT-ICR detection typically requires of the order of 100 or more ions [36]. Thus, low-abundance MS/MS products are more easily detected by QqTOF than by FT-ICR. Furthermore, the experiment is usually simpler with a QqTOF instrument, where "non-experts" can easily acquire relevant data. The experiments done using the home-built 9.4 tesla FT-ICR were much less "routine" and required specialized considerations, as described in the Experimental section. It is almost certain, however, that the latest generation commercial FT-ICR instruments, such as the recently introduced linear ion trap FT-ICR [37], will allow less experienced mass spectrometrists to conduct high resolution tandem MS experiments with little knowledge about the FT-ICR fundamentals. It is also worth noting that some of the low abundant ions of STX and NEO could not be detected by 7 tesla FT-ICR MS (1 s scan time).

\section{Conclusions}

The performance of two types of mass spectrometers, QqTOF and FT-ICR, in their ability to assign elemental formulas from very complex MS/MS spectra has been compared. The product ion mass spectra of two paralytic shellfish poisons were used for comparison because their spectra exhibit an unusually rich variety of product ions. The high peak density results in numerous spectral interferences in the QqTOF MS/MS spectra, mainly due to doublet, triplet, and quadruplet signals from isobaric fragment ion species and from $\mathrm{M}+1$ interferences originating from the coactivated ${ }^{13} \mathrm{C}^{12} \mathrm{C}_{\mathrm{c}-1} \mathrm{MH}^{+}$ions from PSPs. Accordingly, the QqTOF first hit rate for assigning true elemental composition was only 56 and $65 \%$ for STX 
Table 4. The measured masses, elemental formulae and mass measurement uncertainties (ppm) for product ions observed in STX and NEO spectra from commercial 7 Tesla IRMPD FT-ICR and QqTOF CID instruments. Both systems were externally calibrated

\begin{tabular}{|c|c|c|c|c|c|}
\hline $\begin{array}{c}\text { Calculated } \\
\text { mass }\end{array}$ & $\begin{array}{c}\text { Elemental } \\
\text { formula }\end{array}$ & FT-ICR (7 T) & Error (ppm) & QqTOF & Error (ppm) \\
\hline \multicolumn{6}{|c|}{ a. STX } \\
\hline 300.14148 & $\mathrm{C}_{10} \mathrm{H}_{18} \mathrm{~N}_{7} \mathrm{O}_{4}^{+}$ & 300.14147 & 0.0 & 300.1411 & -1.3 \\
\hline 283.11493 & $\mathrm{C}_{10} \mathrm{H}_{15} \mathrm{~N}_{6} \mathrm{O}_{4}^{+}$ & 283.11536 & 1.5 & 283.1160 & 3.8 \\
\hline 282.13091 & $\mathrm{C}_{10} \mathrm{H}_{16} \mathrm{~N}_{7} \mathrm{O}_{3}{ }^{+}$ & 282.13129 & 1.3 & 282.1307 & -0.8 \\
\hline 266.08838 & $\mathrm{C}_{10} \mathrm{H}_{12} \mathrm{~N}_{5} \mathrm{O}_{4}^{+}$ & 266.08875 & 1.4 & 266.0886 & 0.8 \\
\hline 265.10436 & $\mathrm{C}_{10} \mathrm{H}_{13} \mathrm{~N}_{6} \mathrm{O}_{3}{ }^{+}$ & 265.10465 & 1.1 & 265.1040 & -1.4 \\
\hline 241.09313 & $\mathrm{C}_{9} \mathrm{H}_{13} \mathrm{~N}_{4} \mathrm{O}_{4}^{+}$ & 241.09345 & 1.3 & 241.0942 & 4.4 \\
\hline 240.10912 & $\mathrm{C}_{9} \mathrm{H}_{14} \mathrm{~N}_{5} \mathrm{O}_{3}^{+}$ & 240.10954 & 1.8 & 240.1094 & 1.2 \\
\hline 239.12510 & $\mathrm{C}_{9} \mathrm{H}_{15} \mathrm{~N}_{6} \mathrm{O}_{2}^{+}$ & 239.12550 & 1.7 & 239.1251 & 0.0 \\
\hline 222.09855 & $\mathrm{C}_{9} \mathrm{H}_{12} \mathrm{~N}_{5} \mathrm{O}_{2}^{+}$ & 222.09883 & 1.3 & 222.0981 & -2.0 \\
\hline 221.11454 & $\mathrm{C}_{9} \mathrm{H}_{13} \mathrm{~N}_{6} \mathrm{O}^{+}$ & 221.11486 & 1.5 & 221.1145 & -0.2 \\
\hline 204.08799 & $\mathrm{C}_{9} \mathrm{H}_{10} \mathrm{~N}_{5} \mathrm{O}^{+}$ & 204.08829 & 1.5 & 204.0886 & 3.0 \\
\hline 180.07675 & $\mathrm{C}_{8} \mathrm{H}_{10} \mathrm{~N}_{3} \mathrm{O}_{2}{ }^{+}$ & 180.07712 & 2.0 & 180.0833 & 36.4 \\
\hline 179.09274 & $\mathrm{C}_{8} \mathrm{H}_{11} \mathrm{~N}_{4} \mathrm{O}^{+}$ & 179.09302 & 1.6 & 179.0930 & 1.5 \\
\hline 162.06619 & $\mathrm{C}_{8} \mathrm{H}_{8} \mathrm{~N}_{3} \mathrm{O}^{+}$ & 162.06644 & 1.6 & 162.0691 & 18.0 \\
\hline 137.07094 & $\mathrm{C}_{7} \mathrm{H}_{9} \mathrm{~N}_{2} \mathrm{O}^{+}$ & 137.08241 & $1.7^{\mathrm{a}}$ & 137.0801 & 66.8 \\
\hline
\end{tabular}

b. NEO

\begin{tabular}{rlllrr}
316.13639 & $\mathrm{C}_{10} \mathrm{H}_{18} \mathrm{~N}_{7} \mathrm{O}_{5}{ }^{+}$ & 316.13656 & 0.5 & 316.1374 & 3.2 \\
298.12583 & $\mathrm{C}_{10} \mathrm{H}_{16} \mathrm{~N}_{7} \mathrm{O}_{4}^{+}$ & 298.12596 & 0.4 & 298.1264 & 1.9 \\
281.09928 & $\mathrm{C}_{10} \mathrm{H}_{13} \mathrm{~N}_{6} \mathrm{O}_{4}^{+}$ & 281.09954 & 0.9 & 281.1003 & 3.6 \\
263.08872 & $\mathrm{C}_{10} \mathrm{H}_{11} \mathrm{~N}_{6} \mathrm{O}_{3}^{+}$ & 263.08888 & 0.6 & 263.0893 & 2.2 \\
237.10945 & $\mathrm{C}_{9} \mathrm{H}_{13} \mathrm{~N}_{6} \mathrm{O}_{2}^{+}$ & 237.10964 & 0.8 & 237.1102 & 3.2 \\
220.10671 & $\mathrm{C}_{9} \mathrm{H}_{12} \mathrm{~N}_{6} \mathrm{O}^{+}$ & 220.10691 & 0.9 & 220.0970 & -44.1 \\
220.08290 & $\mathrm{C}_{9} \mathrm{H}_{10} \mathrm{~N}_{5} \mathrm{O}_{2}^{+}$ & 220.08314 & 1.1 & 220.0970 & 64.1 \\
207.09889 & $\mathrm{C}_{8} \mathrm{H}_{11} \mathrm{~N}_{6} \mathrm{O}^{+}$ & 207.09907 & $0.9^{\mathrm{a}}$ & 207.0997 & -3.9 \\
195.08765 & $\mathrm{C}_{8} \mathrm{H}_{11} \mathrm{~N}_{4} \mathrm{O}_{2}^{+}$ & 195.08799 & 1.7 & 195.0869 & -10.7 \\
178.08491 & $\mathrm{C}_{8} \mathrm{H}_{10} \mathrm{~N}_{4} \mathrm{O}^{+}$ & 178.08516 & $1.4^{\mathrm{a}}$ & 178.0830 & 14.8 \\
177.07709 & $\mathrm{C}_{8} \mathrm{H}_{9} \mathrm{~N}_{4} \mathrm{O}^{+}$ & 177.07732 & 1.3 & 177.0797 & \\
\hline
\end{tabular}

${ }^{a}$ These numbers were obtained at 100\% IRMPD laser setting. For all other experiments, a laser power setting of $50 \%$ (STX) and $80 \%$ (NEO), respectively, was used (see Experimental).

and NEO product ions, respectively, whereas the FT-ICR experiments yielded first hits in $100 \%$ of all cases. We were able to improve the QqTOF hit rate to $75 \%$ for STX by use of a modified data acquisition protocol, by activating only the monoisotopic precursor ions and thereby largely eliminating the $\mathrm{M}+1{ }^{13} \mathrm{C}^{12} \mathrm{C}_{\mathrm{c}-1}$ interferences. NEO was more difficult because most interferences originate from unique fragment ions rather than from $\mathrm{M}+1{ }^{13} \mathrm{C}^{12} \mathrm{C}_{\mathrm{c}-1}$ interferences. Moreover, the QqTOF hit rate in our experiments was almost $100 \%$ when only the noninterfering species were considered, showing that automated formulas assignments are readily possible for pure samples in regular MS mode or for interference-free MS/MS spectra. It should be emphasized that low-energy CID spectra of synthetic pharmaceutical drugs and their metabolites are usually relatively simple, and automated high-throughput processing of QqTOF accurate mass data generated from these spectra would most likely give excellent hit rates in most cases.

Clearly, there are other factors that need to be included in a complete performance comparison of QqTOF and FT-ICR instruments. For example, practical issues such as the length of time required for obtaining statistically meaningful spectra, the practicality of hy- phenation with ultra-fast chromatography, or the suitability for applications in open access environments are important considerations. These are areas where QqTOF instruments are routinely implemented, for example in pharmaceutical research laboratories. The latest generation FT-ICR instruments, however, where MS/MS experiments can be conducted in the front end, either with a linear quadrupole ion trap or a $\mathrm{Qq}$ arrangement, coupled to an FT-ICR mass analyzer, allow increased compatibility with fast chromatography runs [37]. Several researchers have investigated requirements for hyphenating high-resolution mass spectrometry with modern separation techniques. For example, Grange and coworkers [38] have summarized the requirements for determining elemental compositions for compounds eluting from chromatographic columns. Our study, however, was mainly concerned with very difficult to resolve product ion spectra of high peak densities. For the present MS/MS spectra, which exhibit a variety of isobaric interferences, FT-ICR is required to obtain correct elemental compositions. The required resolving power of $>20,000$ (FWHM) is currently out of reach for QqTOF instruments.

In conclusion, the answer to the question put for- 
ward in the paragraph introducing this article is that the resolving power of QqTOF is not in general sufficient for accurate mass assignments to peaks in very complex MS/MS spectra. For such complex spectra, both high resolving power and mass accuracy are required, as available from FT-ICR instruments.

\section{Acknowledgments}

The authors thank Dr. Michael Chalmers (National High Magnetic Field Laboratory, Tallahassee, FL), Dr. Kevin Crellin and Dr. Robert Williams (Ionspec, Irvine, CA), as well as Dr. Chris Lock (MDS Sciex, Concord, ON) for their support in the FT-ICR and QqTOF analyses. LS acknowledges financial assistance received from the National Sciences and Engineering Research Council of Canada (NSERC) and the NRC's Graduate Student Scholarship Supplement Program (GSSSP). AGM acknowledges support from NSF (CHE-99-09502), Florida State University, and the National High Magnetic Field Laboratory in Tallahassee, FL.

\section{References}

1. Watt, A. P.; Mortishire-Smith, R. J.; Gerhard, U.; Thomas, S. R. Metabolite identification in drug discovery. Curr. Opin. Drug Discov. Dev. 2003, 6, 57-65.

2. Papac, D. I.; Shahrokh, Z. Mass spectrometry innovations in drug discovery and development. Pharm. Res. 2001, 18, 131-145.

3. Hoke, S. H.; Morand, K. L.; Greis, K. D.; Baker, T. R.; Harbol, K. L.; Dobson, R. L. M. Transformations in pharmaceutical research and development, driven by innovations in multidimensional mass spectrometry-based technologies. Int. J. Mass Spectrom. 2001, 212, 135-196.

4. Lee, M. S.; Kerns, E. H. LC/MS applications in drug development. Mass Spectrom. Rev. 1999, 18, 187-279.

5. Kostiainen, R.; Kotiaho, T.; Kuuranne, T.; Auriola, S. Liquid chromatography/atmospheric pressure ionization-mass spectrometry in drug metabolism studies. J. Mass Spectrom. 2003, $38,357-372$

6. Cody, R. B.; Freiser, B. S. High-resolution detection of collision-induced dissociation fragments by Fourier transform mass spectrometry. Anal. Chem. 1982, 54, 1431-1433.

7. Colombo, M.; Sirtori, F. R.; Rizzo, V. A fully automated method for accurate mass determination using high-performance liquid chromatography with a quadrupole/orthogonal acceleration time-of-flight mass spectrometer. Rapid Commun. Mass Spectrom. 2004, 18, 511-517.

8. Fang, L. L.; Demee, M.; Cournoyer, J.; Sierra, T.; Young, C.; Yan, B. Parallel high-throughput accurate mass measurement using a nine-channel multiplexed electrospray liquid chromatography ultraviolet time-of-flight mass spectrometry system. Rapid Commun. Mass Spectrom. 2003, 17, 1425-1432.

9. Belov, M. E.; Anderson, G. A.; Wingerd, M. A.; Udseth, H. R.; Tang, K. Q.; Prior, D. C.; Swanson, K. R.; Buschbach, M. A.; Strittmatter, E. F.; Moore, R. J.; Smith, R. D. An automated high performance capillary liquid chromatography-Fourier transform ion cyclotron resonance mass spectrometer for high-throughput proteomics J. Am. Soc. Mass Spectrom. 2004, $15,212-232$

10. Sleno, L.; Windust, A. J.; Volmer, D. A. Structural study of spirolide marine toxins by mass spectrometry-Part I. Fragmentation pathways of 13-desmethyl spirolide $C$ by collisioninduced dissociation and infrared multiphoton dissociation mass spectrometry. Anal. Bioanal. Chem. 2004, 378, 969-976.
11. Sleno, L.; Windust, A. J.; Volmer, D. A. Structural study of spirolide marine toxins by mass spectrometry-Part II. Characterization of unknown spirolides and related compounds in cultured phytoplankton extract. Anal. Bioanal. Chem. 2004, 378, 977-986.

12. Fiehn, O.; Kopka, J.; Trethewey, R. N.; Willmitzer, L. Identification of uncommon plant metabolites based on calculation of elemental compositions using gas chromatography and quadrupole mass spectrometry. Anal. Chem. 2000, 72, 3573-3580.

13. Eckers, C.; Wolff, J. C.; Haskins, N. J.; Sage, A. B.; Giles, K.; Bateman, R. Accurate mass liquid chromatography/mass spectrometry on orthogonal acceleration time-of-flight mass analyzers using switching between separate sample and reference sprays. 1. Proof of concept. Anal. Chem. 2000, 72, 3683-3688.

14. Charles, L. Flow injection of the lock mass standard for accurate mass measurement in electrospray ionization timeof-flight mass spectrometry coupled with liquid chromatography. Rapid Commun. Mass Spectrom. 2003, 17, 1383-1388.

15. Pelander, A.; Ojanpera, I.; Laks, S.; Rasanen, I.; Vuori, E. Toxicological screening with formula-based metabolite identification by liquid chromatography/time-of-flight mass spectrometry. Anal. Chem. 2003, 75, 5710-5718.

16. Marshall, A. G.; Rodgers, R. P. Petroleomics: The next grand challenge for chemical analysis. Acc. Chem. Res. 2004, 37, 53-59.

17. Stenson, A. C.; Marshall, A. G.; Cooper, W. T. Exact masses and chemical formulas of individual Suwannee River fulvic acids from ultrahigh resolution electrospray Ionization Fourier transform ion cyclotron resonance mass spectra. Anal. Chem. 2003, 75, 1275-1284.

18. Sleno, L.; Volmer, D. A.; Kovacevic, B.; Maksic, Z. B. Gasphase dissociation reactions of protonated saxitoxin and neosaxitoxin. J. Am. Soc. Mass Spectrom. 2004, 15, 462-477.

19. Thompson, C. M.; Richards, D. S.; Fancy, S. A.; Perkins, G. L.; Pullen, F. S.; Thom, C. A comparison of accurate mass techniques for the structural elucidation of fluconazole. Rapid Commun. Mass Spectrom. 2003, 17, 2804-2808.

20. Hau, J.; Stadler, R.; Jenny, T. A.; Fay, L. B. Tandem mass spectrometric accurate mass performance of time-of-flight and Fourier transform ion cyclotron resonance mass spectrometry: A case study with pyridine derivatives. Rapid Commun. Mass Spectrom. 2001, 15, 1840-1848.

21. Bristow, A. W. T.; Webb, K. S. Intercomparison study on accurate mass measurement of small molecules in mass spectrometry. J. Am. Soc. Mass Spectrom. 2003, 14, 1086-1098.

22. Lee, H.-N.; Marshall, A. G. Theoretical maximal precision for mass-to-charge ratio, amplitude, and width measurement in ion-counting mass analyzers. Anal. Chem. 2000, 72, 2256-2260.

23. Gauthier, J. W.; Trautman, T. R.; Jacobson, D. B. Sustained off-resonance irradiation for CAD involving FTMS. CAD technique that emulates infrared multiphoton dissociation. Anal. Chim. Acta 1991, 246, 211-225.

24. McLuckey, S. A.; Goeringer, D. E. Slow heating methods in tandem mass spectrometry. J. Mass Spectrom. 1997, 35, 461-474.

25. Senko, M. W.; Hendrickson, C. L.; Pasa-Tolic, L.; Marto, J. A.; White, F. M.; Guan, S.; Marshall, A. G. Electrospray ionization FT-ICR mass spectrometry at 9.4 tesla. Rapid Commun. Mass Spectrom. 1996, 10, 1824-1828.

26. Senko, M. W.; Hendrickson, C. L.; Emmett, M. R.; Shi, S. D.-H.; Marshall, A. G. External accumulation of ions for enhanced electrospray ionization Fourier transform ion cyclotron resonance mass spectrometry. J. Am. Soc. Mass Spectrom. 1997, 8, 970-976.

27. Chalmers, M. J.; Quinn, J. P.; Blakney, G. T.; Emmett, M. R.; Mischak, H.; Gaskell, S. J.; Marshall, A. G. Liquid chromatography-Fourier transform ion cyclotron resonance mass 
spectrometric characterization of protein kinase C phosphorylation. J. Proteome Res. 2003, 2, 373-382.

28. Chowdhury, S. K.; Katta, V.; Chait, B. T. An electrosprayionization mass-spectrometer with new features. Rapid Commun. Mass Spectrom. 1990, 4, 81-87.

29. Wilcox, B. E.; Hendrickson, C. L.; Marshall, A. G. Improved ion extraction from a linear octopole ion trap: SIMION analysis and experimental demonstration. J. Am. Soc. Mass Spectrom. 2002, 13, 1304-1312.

30. Marshall, A. G.; Verdun, F. R. Fourier Transforms in NMR, Optical, and Mass Spectrometry: A User's Handbook; Elsevier: Amsterdam, 1990.

31. Ledford, E. B., Jr.; Rempel, D. L.; Gross, M. L. Space charge effects in Fourier transform mass spectrometry. Mass calibration. Anal. Chem. 1984, 56, 2744-2748.

32. Shi, S. D. H.; Drader, J. J.; Freitas, M. A.; Hendrickson, C. L.; Marshall, A. G. Comparison and interconversion of the two most common frequency-to-mass calibration functions for Fourier transform ion cyclotron resonance mass spectrometry. Int. J. Mass Spectrom. 2000, 196, 591-598.
33. Marshall, A. G.; Wang, T.-C. L.; Ricca, T. L. Tailored excitation for Fourier transform ion cyclotron resonance mass spectrometry. J. Am. Chem. Soc. 1985, 107, 7893-7897.

34. Guan, S.; Marshall, A. G. Stored waveform inverse Fourier transform (SWIFT) ion excitation in trapped-ion mass spectrometry: Theory and applications. Int. J. Mass Spectrom. Ion Processes 1996, 158, 5-37.

35. Meija, J.; Caruso, J. A. Deconvolution of isobaric interferences in mass spectra. J. Am. Soc. Mass Spectrom. 2004, 15, 654-658.

36. Limbach, P. A.; Grosshans, P. B.; Marshall, A. G. Experimental determination of the number of trapped ions, detection limit, and dynamic range in FT/ICR/MS. Anal. Chem. 1993, 65, 135-140.

37. Olsen, J. V.; Ong, S.-E.; Mann, M. Trypsin cleaves exclusively C-terminal to arginine and lysine residues. Mol. Cell. Proteom. 2004, 3, 608-614.

38. Grange, A. H.; Genicola, F. A.; Sovocool, G. W. Utility of three types of mass spectrometers for determining elemental compositions of ions formed from chromatographically separated compounds. Rapid Commun. Mass Spectrom. 2002, 16, 2356-2369. 